\title{
Hopf bifurcation of a delayed worm model with two latent periods
}

Juan Liu ${ }^{1 *}$ and Zizhen Zhang ${ }^{2}$

${ }^{\text {"Correspondence: }}$ liujuan7216@163.com

${ }^{1}$ School of Science, Bengbu University, Bengbu, China

Full list of author information is available at the end of the article

\section{Springer}

\begin{abstract}
We investigate a delayed epidemic model for the propagation of worm in wireless sensor network with two latent periods. We derive sufficient conditions for local stability of the worm-induced equilibrium of the system and the existence of Hopf bifurcation by regarding different combination of two latent time delays as the bifurcation parameter and analyzing the distribution of roots of the associated characteristic equation. In particular, we investigate the direction and stability of the Hopf bifurcation by means of the normal form theory and center manifold theorem. To verify analytical results, we present numerical simulations. Also, the effect of some influential parameters of sensor network is properly executed so that the oscillations can be reduced and removed from the network.
\end{abstract}

Keywords: Delay; Latent period; Hopf bifurcation; Stability; Wireless sensor network

\section{Introduction}

Coupled with the progress of the digital era and increasing development of various network applications, networks have become more and more popular in our daily life [1-3]. Among the popular networks, the wireless sensor network is one of the most vulnerable to attacks of malicious codes due to its special structure, such as limited capacity and defense capability constraints. Wireless sensor networks are usually made up of hundreds, even thousands, of sensor nodes placed in a hostile or dangerous environment and organized in ad hoc paradigm to monitor the environment where they are not physically safe $[4,5]$. Hence, for upgrading the security in wireless sensor networks, the test on better investigation of malicious codes spreading dynamics is a very crucial subject. For this reason, one of the imperious topics is to formulate reliable mathematical models that are applicable to effectively provide some insights into the characteristics of malicious codes spreading dynamics $[6,7]$ due to the compelling analogies between malicious codes and their biological counterparts. In recent years, some scholars at home and abroad formulated and investigated various mathematical models to study the spread of malicious codes in wireless sensor networks.

Tang and Mark [8] proposed a modified Susceptible-Infected-Recovered (SIR) model by introducing a maintenance mechanism in the sleep mode of wireless sensor networks to characterize the dynamics of the virus spreading process from a single node to the entire network. Unfortunately, Tang and Mark [8] assume that the recovered nodes have permanent immunity, which is not consistent with reality in networks. Because that the

(c) The Author(s) 2019. This article is distributed under the terms of the Creative Commons Attribution 4.0 International License (http://creativecommons.org/licenses/by/4.0/), which permits unrestricted use, distribution, and reproduction in any medium, provided you give appropriate credit to the original author(s) and the source, provide a link to the Creative Commons license, and indicate if changes were made. 
recovered nodes may be infected again by newly emerging viruses. To overcome this defect of the modified SIR model, Feng et al. [9] formulated an improved Susceptible-InfectedRecovered-Susceptible (SIRS) worm propagation model in a wireless sensor network and considered the model communication radius and distributed density of nodes. Zhu et al. [10] developed a delayed SIRS reaction-diffusion model with a state feedback controller to describe the process of malware propagation in mobile wireless sensor networks and studied the Hopf bifurcation of the model. Considering the latent characteristic of malicious codes, Keshri and Mishra [4] proposed a delayed Susceptible-Exposed-InfectiousRecovered (SEIR) to describe the transmission dynamics of malicious signals in wireless sensor network. Vaccination and quarantine are well-known countermeasures in epidemiology. Thus it is interesting and important to extend epidemic models with quarantine and vaccination to study the malicious codes propagation in wireless sensor networks. Mishra and Keshri et al. [11-15] proposed different epidemic models with vaccination to study the attacking behavior of malicious codes in wireless sensor networks. Ojha et al. [1618] formulated different models with quarantine to depict worm propagation behavior in wireless sensor network. There are also some dynamical models with both vaccination and quarantine $[19,20]$ and other models $[21-24]$ to model the dynamics of malicious codes in wireless sensor networks. It should be pointed out that most of the models considering the latent state above assume that all the malicious codes in the wireless sensor network have the same latent period. However, different types of malicious codes are available in the digital environment, and they require no any human intervention or infrastructure network for transmission. Based on this consideration, Ojha et al. [25] proposed the following model for the transmission of worm in wireless sensor with two latent periods:

$$
\left\{\begin{array}{l}
\frac{d S(t)}{d t}=b-\beta S(t) I(t)-\sigma S(t), \\
\frac{d E_{1}(t)}{d t}=p \beta S(t) I(t)-\left(\alpha_{1}+\sigma\right) E_{1}(t), \\
\frac{d E_{2}(t)}{d t}=q \beta S(t) I(t)-\left(\alpha_{2}+\sigma\right) E_{2}(t), \\
\frac{d I(t)}{d t}=\alpha_{1} E_{1}(t)+\alpha_{2} E_{2}(t)-(\gamma+\sigma) I(t), \\
\frac{d R(t)}{d t}=\gamma I(t)-\sigma R(t),
\end{array}\right.
$$

where $S(t), E_{1}(t), E_{2}(t)$, and $I(t)$ denote the numbers of the susceptible infected class of short latent period, the infected class of long latent period, the infectious, and the recovered nodes at time $t$, respectively. More parameters are listed in Table 1. Ojha et al. [25] studied the stability of system (1).

Table 1 Parameters and their meanings

\begin{tabular}{ll}
\hline Parameter & Description \\
\hline$b$ & The constant recruitment to susceptible nodes \\
$\beta$ & Rate at which susceptible nodes become infected \\
$p$ & The amount from susceptible nodes to the first category exposed nodes \\
$q$ & The amount from susceptible nodes to the second category exposed nodes \\
$\alpha_{1}$ & The rate the first exposed category of nodes become infectious \\
$\alpha_{2}$ & The rate the second exposed category of nodes become infectious \\
$\gamma$ & Recovery rate of the infectious nodes \\
$\sigma$ & Per capita death rate \\
\hline
\end{tabular}


However, as stated in [26], one of the typical features for the malicious codes in networks is their latency. There is usually a delay from the time the $E_{1}$ and $E_{2}$ nodes are infected to the time they become infectious due to the intrinsic latent period of worms. On the other hand, delay differential equations exhibit much more complicated dynamics than ordinary differential equations since a time delay can cause the Hopf bifurcation phenomenon and changes the behavior of a dynamical system from stable focus to limit cycle [27-32]. Hence the study of the complex dynamical behaviors of system (2) with time delay, especially the Hopf bifurcation, also is very important for the transmission and controlling of the worms. Thus we incorporate the latent delay of the two categories of worms into system (1) and investigate the following delayed system:

$$
\left\{\begin{array}{l}
\frac{d S(t)}{d t}=b-\beta S(t) I(t)-\sigma S(t), \\
\frac{d E_{1}(t)}{d t}=p \beta S(t) I(t)-\sigma E_{1}(t)-\alpha_{1} E_{1}\left(t-\tau_{1}\right), \\
\frac{d E_{2}(t)}{d t}=q \beta S(t) I(t)-\sigma E_{2}(t)-\alpha_{2} E_{2}\left(t-\tau_{2}\right), \\
\frac{d I(t)}{d t}=\alpha_{1} E_{1}\left(t-\tau_{1}\right)+\alpha_{2} E_{2}\left(t-\tau_{2}\right)-(\gamma+\sigma) I(t), \\
\frac{d R(t)}{d t}=\gamma I(t)-\sigma R(t),
\end{array}\right.
$$

where $\tau_{1}$ is the latent period of the first category of worm transmit in the wireless sensor network, and $\tau_{2}$ is the latent period of the second category of worm transmit in the wireless sensor network. We may see that the first four equations in system (2) are independent of the fifth equation, and therefore the fifth equation can be omitted. So we will discuss the following reduced system:

$$
\left\{\begin{array}{l}
\frac{d S(t)}{d t}=b-\beta S(t) I(t)-\sigma S(t), \\
\frac{d E_{1}(t)}{d t}=p \beta S(t) I(t)-\sigma E_{1}(t)-\alpha_{1} E_{1}\left(t-\tau_{1}\right), \\
\frac{d E_{2}(t)}{d t}=q \beta S(t) I(t)-\sigma E_{2}(t)-\alpha_{2} E_{2}\left(t-\tau_{2}\right), \\
\frac{d I(t)}{d t}=\alpha_{1} E_{1}\left(t-\tau_{1}\right)+\alpha_{2} E_{2}\left(t-\tau_{2}\right)-(\gamma+\sigma) I(t) .
\end{array}\right.
$$

The structure of this paper is as follows. The local stability of the worm induced equilibrium and existence of Hopf bifurcation are discussed by choosing different combination of $\tau_{1}$ and $\tau_{2}$ as the bifurcation parameter in Sect. 2. We investigate the direction and stability of the Hopf bifurcation at the worm-induced equilibrium when $\tau_{2}>0$ and $\tau_{1} \in\left(0, \tau_{10}\right)$ by using the normal form theory and center manifold theorem. To verify the obtained results and some important parameter effects, we accomplish some numerical simulations in Sect. 4. We conclude the paper in Sect. 5.

\section{Local stability and existence of Hopf bifurcation}

According to the analysis in [25], we can conclude that system (3) has a worm-induced equilibrium $E_{*}\left(S_{*}, E_{1 *}, E_{2 *}, I_{*}\right)$, where

$$
\begin{aligned}
& S_{*}=\frac{b}{\sigma R_{0}}, \quad E_{1 *}=\frac{p b}{\alpha_{1}+\sigma}\left(\frac{R_{0}-1}{R_{0}}\right), \\
& E_{2 *}=\frac{(1-p) b}{\alpha_{2}+\sigma}\left(\frac{R_{0}-1}{R_{0}}\right), \quad I_{*}=\frac{\sigma}{\beta}\left(R_{0}-1\right),
\end{aligned}
$$


where

$$
R_{0}=\frac{\beta b}{\sigma(\gamma+\sigma)}\left[\frac{p \alpha_{1}}{\alpha_{1}+\sigma}+\frac{q \alpha_{2}}{\alpha_{2}+\sigma}\right], \quad p+q=1
$$

The Jacobian matrix of system (3) evaluated at $E_{*}$ is

$$
J_{E_{*}}=\left(\begin{array}{cccc}
\alpha_{11} & 0 & 0 & \alpha_{14} \\
\alpha_{21} & \alpha_{22}+\beta_{22} e^{-\tau_{1}} & 0 & \alpha_{24} \\
\alpha_{31} & 0 & \alpha_{33}+\gamma_{33} e^{-\lambda \tau_{2}} & \alpha_{34} \\
0 & \beta_{42} e^{-\tau_{1}} & \gamma_{43} e^{-\lambda \tau_{2}} & \alpha_{44}
\end{array}\right) \text {, }
$$

where

$$
\begin{aligned}
& \alpha_{11}=-\left(\beta I_{*}+\sigma\right), \quad \alpha_{14}=-\beta S_{*}, \\
& \alpha_{21}=p \beta I_{*}, \quad \alpha_{22}=-\sigma, \quad \alpha_{24}=p \beta S_{*}, \\
& \alpha_{31}=q \beta I_{*}, \quad \alpha_{33}=-\sigma, \quad \alpha_{34}=q \beta S_{*}, \quad \alpha_{44}=-(\gamma+\sigma), \\
& \beta_{22}=-\alpha_{1}, \quad \beta_{42}=\alpha_{1}, \quad \gamma_{33}=-\alpha_{2}, \quad \gamma_{43}=\alpha_{2} .
\end{aligned}
$$

The characteristic equation associated with system (3) at the worm-induced equilibrium $E_{*}$ is

$$
\begin{aligned}
\lambda^{4} & +m_{3} \lambda^{3}+m_{2} \lambda^{2}+m_{1} \lambda+m_{0} \\
& +\left(n_{3} \lambda^{3}+n_{2} \lambda^{2}+n_{1} \lambda+n_{0}\right) e^{-\lambda \tau_{1}} \\
& +\left(p_{3} \lambda^{3}+p_{2} \lambda^{2}+p_{1} \lambda+p_{0}\right) e^{-\lambda \tau_{2}} \\
& +\left(q_{2} \lambda^{2}+q_{1} \lambda+q_{0}\right) e^{-\lambda\left(\tau_{1}+\tau_{2}\right)}=0
\end{aligned}
$$

where

$$
\begin{aligned}
m_{0}= & \alpha_{11} \alpha_{22} \alpha_{33} \alpha_{44}, \\
m_{1}= & -\left[\alpha_{11} \alpha_{22}\left(\alpha_{33}+\alpha_{44}\right)+\alpha_{33} \alpha_{44}\left(\alpha_{11}+\alpha_{22}\right)\right], \\
m_{2}= & \alpha_{11} \alpha_{22}+\alpha_{33} \alpha_{44}+\left(\alpha_{11}+\alpha_{22}\right)\left(\alpha_{33}+\alpha_{44}\right), \\
m_{3}= & -\left(\alpha_{11}+\alpha_{22}+\alpha_{33}+\alpha_{44}\right), \\
n_{0}= & \alpha_{11} \alpha_{33}\left(\alpha_{44} \beta_{22}-\alpha_{24} \beta_{42}\right)+\alpha_{14} \alpha_{21} \alpha_{33} \beta_{42}, \\
n_{1}= & \alpha_{24} \beta_{42}\left(\alpha_{11}+\alpha_{33}\right)-\alpha_{14} \alpha_{21} \beta_{42} \\
& -\beta_{22}\left(\alpha_{11} \alpha_{33}+\alpha_{11} \alpha_{44}+\alpha_{33} \alpha_{44}\right), \\
n_{2}= & \beta_{22}\left(\alpha_{11}+\alpha_{33}+\alpha_{44}\right)-\alpha_{24} \beta_{42}, \\
p_{0}= & \alpha_{22} \gamma_{43}\left(\alpha_{14} \alpha_{31}-\alpha_{11} \alpha_{34}\right)+\alpha_{11} \alpha_{22} \alpha_{44} \gamma_{33}, \\
p_{1}= & \alpha_{34} \gamma_{43}\left(\alpha_{11}+\alpha_{22}\right)-\alpha_{14} \alpha_{31} \gamma_{43} \\
& -\gamma_{33}\left(\alpha_{11} \alpha_{22}+\alpha_{11} \alpha_{44}+\alpha_{22} \alpha_{44}\right),
\end{aligned}
$$




$$
\begin{aligned}
p_{2}= & \gamma_{33}\left(\alpha_{11}+\alpha_{22}+\alpha_{44}\right)-\alpha_{34} \gamma_{43}, \quad p_{3}=-\gamma_{33}, \\
q_{0}= & \beta_{22} \gamma_{43}\left(\alpha_{14} \alpha_{31}-\alpha_{11} \alpha_{34}\right)+\beta_{42} \gamma_{33}\left(\alpha_{14} \alpha_{21}-\alpha_{11} \alpha_{24}\right) \\
& +\alpha_{11} \alpha_{44} \beta_{22} \gamma_{33}, \\
& q_{1}=\alpha_{24} \beta_{42} \gamma_{33}+\alpha_{34} \beta_{22} \gamma_{43}-\beta_{22} \gamma_{33}\left(\alpha_{11}+\alpha_{44}\right), \quad q_{2}=\beta_{22} \gamma_{33} .
\end{aligned}
$$

Case $1 \tau=0$. Equation (4) becomes

$$
\lambda^{4}+m_{13} \lambda^{3}+m_{12} \lambda^{2}+m_{11} \lambda+m_{10}=0,
$$

where

$$
\begin{array}{ll}
m_{10}=m_{0}+n_{0}+p_{0}+q_{0}, & m_{11}=m_{1}+n_{1}+p_{1}+q_{1}, \\
m_{12}=m_{2}+n_{2}+p_{2}+q_{2}, & m_{13}=m_{3}+n_{3}+p_{3} .
\end{array}
$$

Obviously, $m_{13}=\alpha_{1}+\alpha_{2}+\beta I_{*}+\gamma+4 \sigma>0$. Based on the Hurwitz criterion, we have the following results.

Lemma 1 If condition $\left(H_{1}\right)$ holds, that is, $m_{10}>0, m_{12} m_{13}>m_{11}$, and $m_{11} m_{12} m_{13}>m_{12}^{2}+$ $m_{10} m_{13}^{2}$, then system (3) is locally asymptotically stable when $\tau=0$.

Case $2 \tau_{1}>0, \tau_{2}=0$. Equation (4) reduces to

$$
\lambda^{4}+m_{23} \lambda^{3}+m_{22} \lambda^{2}+m_{21} \lambda+m_{20}+\left(n_{23} \lambda^{3}+n_{22} \lambda^{2}+n_{21} \lambda+n_{20}\right) e^{-\lambda \tau_{1}}=0
$$

with

$$
\begin{aligned}
& m_{20}=m_{0}+p_{0}, \quad m_{21}=m_{1}+p_{1}, \quad m_{22}=m_{2}+p_{2}, \quad m_{23}=m_{3}+p_{3}, \\
& n_{20}=n_{0}+q_{0}, \quad n_{21}=n_{1}+q_{1}, \quad n_{22}=n_{2}+q_{2}, \quad n_{23}=n_{3} .
\end{aligned}
$$

Let $\lambda=i \omega_{1}\left(\omega_{1}>0\right)$ be a root of Eq. (6). Substituting it into Eq. (6) and separating the real and imaginary parts, we get

$$
\left\{\begin{array}{l}
\left(n_{21} \omega_{1}-n_{23} \omega_{1}^{3}\right) \sin \tau_{1} \omega_{1}+\left(n_{20}-n_{22} \omega_{1}^{2}\right) \cos \tau_{1} \omega_{1}=m_{22} \omega_{1}^{2}-\omega_{1}^{4}-m_{20} \\
\left(n_{21} \omega_{1}-n_{23} \omega_{1}^{3}\right) \cos \tau_{1} \omega_{1}-\left(n_{20}-n_{22} \omega_{1}^{2}\right) \sin \tau_{1} \omega_{1}=m_{23} \omega_{1}^{3}-m_{21} \omega_{1}
\end{array}\right.
$$

which implies

$$
\omega_{1}^{8}+h_{23} \omega_{1}^{6}+h_{22} \omega_{1}^{4}+h_{21} \omega_{1}^{2}+h_{20}=0
$$

where

$$
\begin{aligned}
& h_{20}=m_{20}^{2}-n_{20}^{2}, \\
& h_{21}=m_{21}^{2}-2 m_{20} m_{22}-n_{21}^{2}+2 n_{20} n_{22},
\end{aligned}
$$




$$
\begin{aligned}
& h_{22}=m_{22}^{2}+2 m_{20}-2 m_{21} m_{23}+2 n_{21} n_{23}-n_{22}^{2}, \\
& h_{23}=m_{23}^{2}-2 m_{22}-n_{23}^{2} .
\end{aligned}
$$

Let $\omega_{1}^{2}=v_{1}$. Then Eq. (7) becomes

$$
v_{1}^{4}+h_{23} v_{1}^{3}+h_{22} v_{1}^{2}+h_{21} v_{1}+h_{20}=0
$$

Define

$$
\begin{aligned}
& g_{21}\left(v_{1}\right)=v_{1}^{4}+h_{23} v_{1}^{3}+h_{22} v_{1}^{2}+h_{21} v_{1}+h_{20} \\
& \zeta_{20}=\frac{1}{2} h_{22}-\frac{3}{16} h_{23}^{2}, \quad \eta_{20}=\frac{1}{32} h_{23}^{2}-\frac{1}{8} h_{22} h_{23}+h_{21}, \\
& \alpha_{20}=\left(\frac{\eta_{20}}{2}\right)^{2}+\left(\frac{\zeta_{20}}{3}\right), \quad \beta_{20}=-\frac{1}{2}+\frac{\sqrt{3}}{2} i \\
& x_{21}=\sqrt{-\frac{\eta_{20}}{2}+\sqrt[3]{\alpha_{20}}}+\sqrt[3]{-\frac{\eta_{20}}{2}-\sqrt{\alpha_{20}}}, \\
& x_{22}=\beta_{20} \sqrt{-\frac{\eta_{20}}{2}+\sqrt[3]{\alpha_{20}}}+\beta_{20}^{2} \sqrt[3]{-\frac{\eta_{20}}{2}-\sqrt{\alpha_{20}}} \\
& x_{23}=\beta_{20}^{2} \sqrt{-\frac{\eta_{20}}{2}+\sqrt[3]{\alpha_{20}}}+\beta_{20} \sqrt[3]{-\frac{\eta_{20}}{2}-\sqrt{\alpha_{20}}} \\
& v_{1 i}=y_{2 i}-\frac{3 h_{23}}{4}, \quad i=1,2,3 .
\end{aligned}
$$

Based on the distribution of the roots of Eq. (8) in [33], we have the following results.

Lemma 2 For Eq. (8), we have:

(i) if $h_{20}<0$, then Eq. (8) has at least one positive root;

(ii) if $h_{20} \geq 0$ and $\alpha_{20} \geq 0$, then Eq. (8) has positive roots if only if $v_{11}>0$ and $g_{21}\left(v_{11}\right)<0$;

(iii) if $h_{20} \geq 0$ and $\alpha_{20}<0$, then $E q$. (8) has positive roots if only if there exists at least one $v_{1 *} \in\left\{v_{11}, v_{12}, v_{13}\right\}$ such that $v_{1 *}>0$ and $g_{21}\left(v_{1 *}\right)>0$.

We further suppose that

$\left(H_{21}\right)$ : the coefficients $h_{20}, h_{21}, h_{22}$, and $h_{23}$ in $g_{21}\left(v_{1}\right)$ satisfy one of the conditions

(a) $h_{20}<0$,

(b) $h_{20} \geq 0, \alpha_{20} \geq 0, v_{11}>0$, and $g_{1}\left(v_{11}\right)<0$,

(c) $h_{20} \geq 0$, and there exists at least one $v_{1 *} \in\left\{v_{11}, v_{12}, v_{13}\right\}$ such that $v_{1 *}>0$ and $g_{21}\left(v_{1 *}\right)>0, \alpha_{20}<0$.

Thus we can conclude that there exists a positive root $\omega_{10}$ of Eq. (7) such that Eq. (6) has a pair of purely imaginary roots $\pm i \omega_{10}$. For $\omega_{10}$, we have

$$
\tau_{10}=\frac{1}{\omega_{10}} \times \arccos \left\{\frac{P_{21}\left(\omega_{10}\right)}{Q_{21}\left(\omega_{10}\right)}\right\},
$$

where

$$
\begin{aligned}
P_{21}\left(\omega_{10}\right)= & \left(n_{22}-m_{23} n_{23}\right) \omega_{10}^{6}+\left(m_{23} n_{21}+m_{21} n_{23}-n_{20}-m_{22} n_{22}\right) \omega_{10}^{4} \\
& +\left(m_{22} n_{20}+m_{20} n_{22}-m_{21} n_{21}\right) \omega_{10}^{2}-m_{20} n_{20} \\
Q_{21}\left(\omega_{10}\right)= & n_{23}^{2} \omega_{10}^{6}+\left(n_{22}^{2}-2 n_{21} n_{23}\right) \omega_{10}^{4}+\left(n_{21}^{2}-2 n_{20} n_{22}\right) \omega_{10}^{2}+n_{20}^{2} .
\end{aligned}
$$


Differentiating both sides of Eq. (4) with respect to $\tau_{1}$ yields

$$
\left[\frac{d \lambda}{d \tau_{1}}\right]^{-1}=-\frac{4 \lambda^{3}+3 m_{23} \lambda^{2}+2 m_{22} \lambda+m_{21}}{\lambda\left(\lambda^{4}+m_{23} \lambda^{3}+m_{22} \lambda^{2}+m_{21} \lambda+m_{20}\right)}+\frac{3 n_{23} \lambda^{2}+2 n_{22} \lambda+n_{21}}{\lambda\left(n_{23} \lambda^{3}+n_{22} \lambda^{2}+n_{21} \lambda+n_{20}\right)}-\frac{\tau}{\lambda} .
$$

Hence we obtain

$$
\operatorname{Re}\left[\frac{d \lambda}{d \tau_{1}}\right]_{\tau_{1}=\tau_{10}}^{-1}=\frac{g_{21}^{\prime}\left(v_{10}\right)}{Q_{21}\left(\omega_{10}\right)}
$$

where $g_{21}\left(v_{1}\right)=v_{1}^{4}+h_{23} v_{1}^{3}+h_{22} v_{1}^{2}+h_{21} v_{1}+h_{20}$.

Clearly, if condition

$\left(H_{22}\right): g_{21}^{\prime}\left(v_{10}\right) \neq 0$

is satisfied, then $\operatorname{Re}\left[\frac{d \lambda}{d \tau_{1}}\right]_{\tau_{1}=\tau_{10}}^{-1} \neq 0$. Based on the previous discussion and the Hopf bifurcation theorem in [34], we can obtain the following results.

Theorem 1 For system (3), if conditions $\left(H_{1}\right),\left(H_{21}\right)$, and $\left(H_{22}\right)$ hold, then system (3) is locally asymptotically stable when $\tau_{1} \in\left[0, \tau_{10}\right)$; system (3) undergoes a Hopf bifurcation at the worm-induced equilibrium $E_{*}$ when $\tau_{1}=\tau_{10}$, and a family of periodic solutions bifurcate from the worm-induced equilibrium $E_{*} ; \tau_{10}$ is defined as in $E q$. (10).

Case $3 \tau_{1}=0, \tau_{2}>0$. Equation (4) becomes

$$
\lambda^{4}+m_{33} \lambda^{3}+m_{32} \lambda^{2}+m_{31} \lambda+m_{30}+\left(p_{33} \lambda^{3}+p_{32} \lambda^{2}+p_{31} \lambda+p_{30}\right) e^{-\lambda \tau_{2}}=0
$$

with

$$
\begin{aligned}
& m_{30}=m_{0}+n_{0}, \quad m_{31}=m_{1}+n_{1}, \quad m_{32}=m_{2}+n_{2}, \quad m_{33}=m_{3}+n_{3}, \\
& p_{30}=p_{0}+q_{0}, \quad p_{31}=p_{1}+q_{1}, \quad p_{32}=p_{2}+q_{2}, \quad p_{33}=p_{3} .
\end{aligned}
$$

Let $\lambda=i \omega_{2}\left(\omega_{2}>0\right)$ be a root of Eq. (11). Substituting it into Eq. (11) and separating the real and imaginary parts, we get

$$
\left\{\begin{array}{l}
\left(p_{31} \omega_{2}-p_{33} \omega_{2}^{3}\right) \sin \tau_{2} \omega_{2}+\left(p_{30}-p_{32} \omega_{2}^{2}\right) \cos \tau_{2} \omega_{2}=m_{32} \omega_{2}^{2}-\omega_{2}^{4}-m_{30} \\
\left(p_{31} \omega_{2}-p_{33} \omega_{2}^{3}\right) \cos \tau_{2} \omega_{2}-\left(p_{30}-p_{32} \omega_{2}^{2}\right) \sin \tau_{2} \omega_{2}=m_{33} \omega_{2}^{3}-m_{31} \omega_{2}
\end{array}\right.
$$

which leads to

$$
\omega_{2}^{8}+h_{33} \omega_{2}^{6}+h_{32} \omega_{2}^{4}+h_{31} \omega_{2}^{2}+h_{20}=0
$$

where

$$
\begin{aligned}
& h_{30}=m_{30}^{2}-p_{30}^{2}, \\
& h_{31}=m_{31}^{2}-2 m_{30} m_{32}-p_{31}^{2}+2 p_{30} p_{32}, \\
& h_{32}=m_{32}^{2}+2 m_{30}-2 m_{31} m_{33}+2 p_{31} p_{33}-p_{32}^{2}, \\
& h_{33}=m_{33}^{2}-2 m_{32}-p_{33}^{2} .
\end{aligned}
$$


Let $\omega_{2}^{2}=v_{1}$. Then Eq. (12) becomes

$$
v_{2}^{4}+h_{33} v_{2}^{3}+h_{32} v_{2}^{2}+h_{31} v_{2}+h_{30}=0
$$

Define

$$
\begin{aligned}
& g_{31}\left(v_{2}\right)=v_{2}^{4}+h_{33} v_{2}^{3}+h_{32} v_{2}^{2}+h_{31} v_{2}+h_{30} \\
& \zeta_{30}=\frac{1}{2} h_{32}-\frac{3}{16} h_{33}^{2}, \quad \eta_{30}=\frac{1}{32} h_{33}^{2}-\frac{1}{8} h_{32} h_{33}+h_{31}, \\
& \alpha_{30}=\left(\frac{\eta_{30}}{2}\right)^{2}+\left(\frac{\zeta_{30}}{3}\right), \quad \beta_{30}=-\frac{1}{2}+\frac{\sqrt{3}}{2} i \\
& x_{31}=\sqrt{-\frac{\eta_{30}}{2}+\sqrt[3]{\alpha_{30}}}+\sqrt[3]{-\frac{\eta_{30}}{2}-\sqrt{\alpha_{30}}} \\
& x_{32}=\beta_{30} \sqrt{-\frac{\eta_{30}}{2}+\sqrt[3]{\alpha_{30}}}+\beta_{30}^{2} \sqrt[3]{-\frac{\eta_{30}}{2}-\sqrt{\alpha_{30}}} \\
& x_{33}=\beta_{30}^{2} \sqrt{-\frac{\eta_{30}}{2}+\sqrt[3]{\alpha_{30}}}+\beta_{30} \sqrt[3]{-\frac{\eta_{30}}{2}-\sqrt{\alpha_{30}}} \\
& v_{2 i}=x_{3 i}-\frac{3 h_{33}}{4}, \quad i=1,2,3 .
\end{aligned}
$$

Based on the distribution of the roots of Eq. (13), we have the following results.

Lemma 3 For Eq. (13), we have:

(i) if $h_{30}<0$, then Eq. (13) has at least one positive root;

(ii) if $h_{30} \geq 0$ and $\alpha_{30} \geq 0$, then Eq. (13) has positive roots if only if $v_{21}>0$ and $g_{22}\left(v_{21}\right)<0$;

(iii) if $h_{30} \geq 0$ and $\alpha_{30}<0$, then Eq. (13) has positive roots if only if there exists at least one $v_{2 *} \in\left\{v_{21}, v_{22}, v_{23}\right\}$ such that $v_{2 *}>0$ and $g_{22}\left(v_{2 *}\right)>0$.

We further suppose that

$\left(H_{31}\right)$ : the coefficients $h_{30}, h_{31}, h_{32}$, and $h_{33}$ in $g_{22}\left(v_{2}\right)$ satisfy one of the conditions

(a') $h_{30}<0$,

$\left(b^{\prime}\right) h_{30} \geq 0, \alpha_{30} \geq 0, v_{21}>0$, and $g_{22}\left(v_{21}\right)<0$,

(c') $h_{30} \geq 0$,

and there exists at least one $v_{2 *} \in\left\{v_{21}, v_{22}, v_{23}\right\}$ such that $v_{2 *}>0$ and $g_{22}\left(v_{2 *}\right)>0$, $\alpha_{30}<0$.

Then we know that there exists a positive root $\omega_{20}$ of Eq. (12) such that Eq. (11) has a pair of purely imaginary roots $\pm i \omega_{20}$. For $\omega_{20}$, we have

$$
\tau_{20}=\frac{1}{\omega_{20}} \times \arccos \left\{\frac{P_{31}\left(\omega_{20}\right)}{Q_{31}\left(\omega_{20}\right)}\right\},
$$

where

$$
\begin{aligned}
P_{31}\left(\omega_{10}\right)= & \left(p_{32}-m_{33} p_{33}\right) \omega_{20}^{6}+\left(m_{33} p_{31}+m_{31} p_{33}-p_{30}-m_{32} p_{32}\right) \omega_{20}^{4} \\
& +\left(m_{32} p_{30}+m_{30} p_{32}-m_{31} p_{31}\right) \omega_{20}^{2}-m_{30} p_{30} \\
Q_{31}\left(\omega_{10}\right)= & p_{33}^{2} \omega_{20}^{6}+\left(p_{32}^{2}-2 p_{31} p_{33}\right) \omega_{20}^{4}+\left(p_{31}^{2}-2 p_{30} p_{32}\right) \omega_{20}^{2}+p_{30}^{2}
\end{aligned}
$$


Similarly as in Case 2, we can obtain

$$
\operatorname{Re}\left[\frac{d \lambda}{d \tau_{2}}\right]_{\tau_{2}=\tau_{20}}^{-1}=\frac{g_{22}^{\prime}\left(v_{20}\right)}{Q_{31}\left(\omega_{20}\right)}
$$

where $g_{22}\left(v_{2}\right)=v_{2}^{4}+h_{33} v_{2}^{3}+h_{32} v_{2}^{2}+h_{31} v_{2}+h_{30}$.

Thus, if condition

$$
\left(H_{32}\right): g_{22}^{\prime}\left(v_{20}\right) \neq 0
$$

holds, then $\operatorname{Re}\left[\frac{d \lambda}{d \tau_{2}}\right]_{\tau_{1}=\tau_{20}}^{-1} \neq 0$. In conclusion, we have the following results.

Theorem 2 For system (3), if conditions $\left(H_{1}\right),\left(H_{31}\right)$, and $\left(H_{32}\right)$ hold, then system (3) is locally asymptotically stable when $\tau_{2} \in\left[0, \tau_{20}\right)$; system (3) undergoes a Hopf bifurcation at the worm-induced equilibrium $E_{*}$ when $\tau_{2}=\tau_{20}$, and a family of periodic solutions bifurcate from the worm-induced equilibrium $E_{*} ; \tau_{20}$ is defined as in Eq. (15).

Case $4 \tau_{1}>0, \tau_{2} \in\left(0, \tau_{20}\right)$. Let $\lambda=i \omega_{11}\left(\omega_{11}>0\right)$ be the root of Eq. (4). Then $\omega_{11}$ must satisfy the following form:

$$
\left\{\begin{array}{l}
M_{41}\left(\omega_{11}\right) \sin \tau_{1} \omega_{11}+M_{42}\left(\omega_{11}\right) \cos \tau_{1} \omega_{11}=M_{43}\left(\omega_{11}\right) \\
M_{41}\left(\omega_{11}\right) \cos \tau_{1} \omega_{11}-M_{42}\left(\omega_{11}\right) \sin \tau_{1} \omega_{11}=M_{44}\left(\omega_{11}\right)
\end{array}\right.
$$

where

$$
\begin{aligned}
M_{41}\left(\omega_{11}\right)= & n_{1} \omega_{11}-n_{3} \omega_{11}^{3}+q_{1} \omega_{11} \cos \tau_{2} \omega_{11}-\left(q_{0}-q_{2} \omega_{11}^{2}\right) \sin \tau_{2} \omega_{11} \\
M_{42}\left(\omega_{11}\right)= & n_{0}-n_{2} \omega_{11}^{2}+q_{1} \omega_{11} \sin \tau_{2} \omega_{11}+\left(q_{0}-q_{2} \omega_{11}^{2}\right) \cos \tau_{2} \omega_{11} \\
M_{43}\left(\omega_{11}\right)= & m_{2} \omega_{11}^{2}-\omega_{11}^{4}-m_{0}-\left(p_{1} \omega_{11}-p_{3} \omega_{11}^{3}\right) \sin \tau_{2} \omega_{11} \\
& -\left(p_{0}-p_{2} \omega_{11}^{2}\right) \cos \tau_{2} \omega_{11} \\
M_{44}\left(\omega_{11}\right)= & m_{3} \omega_{11}^{3}-m_{1} \omega_{11}-\left(p_{1} \omega_{11}-p_{3} \omega_{11}^{3}\right) \cos \tau_{2} \omega_{11} \\
& +\left(p_{0}-p_{2} \omega_{11}^{2}\right) \sin \tau_{2} \omega_{11}
\end{aligned}
$$

Thus we obtain the following equation with respect to $\omega_{11}$ :

$$
M_{43}^{2}\left(\omega_{11}\right)+M_{44}^{2}\left(\omega_{11}\right)-M_{41}^{2}\left(\omega_{11}\right)-M_{42}^{2}\left(\omega_{11}\right)=0 .
$$

Next, we suppose that condition $\left(H_{41}\right)$ holds, that is, Eq. (16) has at least one positive root $\omega_{1}^{*}$. Thus Eq. (4) has a pair of purely imaginary roots $\pm i \omega_{1}^{*}$. For $\omega_{1}^{*}$, we have:

$$
\tau_{1}^{*}=\frac{1}{\omega_{1}^{*}} \times \arccos \left\{\frac{M_{41}\left(\omega_{1}^{*}\right) \times M_{44}\left(\omega_{1}^{*}\right)+M_{42}\left(\omega_{1}^{*}\right) \times M_{43}\left(\omega_{1}^{*}\right)}{M_{41}^{2}\left(\omega_{1}^{*}\right)+M_{42}^{2}\left(\omega_{1}^{*}\right)}\right\} .
$$

Differentiating both sides of Eq. (4) with respect to $\tau_{1}$, we obtain

$$
\left[\frac{d \lambda}{d \tau_{1}}\right]^{-1}=\frac{P_{41}(\lambda)}{Q_{41}(\lambda)}-\frac{\tau_{1}}{\lambda}
$$


with

$$
\begin{aligned}
P_{41}(\lambda)= & 4 \lambda^{3}+3 m_{3} \lambda^{2}+2 m_{2} \lambda+m_{1}+\left(3 n_{3} \lambda^{2}+2 n_{2} \lambda+n_{1}\right) e^{-\lambda \tau_{1}} \\
& -\left[\tau_{2} p_{3} \lambda^{3}-\left(3 p_{3}-\tau_{2} p_{2}\right) \lambda^{2}-\left(2 p_{2}-\tau_{2} p_{1}\right) \lambda-p_{1}+\tau_{2} p_{0}\right] e^{-\lambda \tau_{2}} \\
& -\left[\tau_{2} q_{2} \lambda^{2}-\left(2 q_{2}-\tau_{2} q_{1}\right) \lambda-q_{1}+\tau_{2} q_{0}\right] e^{-\lambda\left(\tau_{1}+\tau_{2}\right)}, \\
Q_{41}(\lambda)= & \lambda\left(n_{3} \lambda^{3}+n_{2} \lambda^{2}+n_{1} \lambda+n_{0}\right) e^{-\lambda \tau_{1}}+\lambda\left(q_{2} \lambda^{2}+q_{1} \lambda+q_{0}\right) e^{-\lambda\left(\tau_{1}+\tau_{2}\right)} .
\end{aligned}
$$

Further, we have

$$
\operatorname{Re}\left[\frac{d \lambda}{d \tau_{1}}\right]_{\tau_{1}=\tau_{1}^{*}}^{-1}=\frac{U_{41} V_{41}+U_{42} V_{42}}{V_{41}^{2}+V_{42}^{2}}
$$

where

$$
\begin{aligned}
U_{41}= & {\left[2 n_{2} \omega_{1}^{*}+\left(2 q_{2}-\tau_{2} q_{1}\right) \omega_{1}^{*} \cos \tau_{2} \omega_{1}^{*}\right.} \\
& \left.-\left(\tau_{2} q_{2}\left(\omega_{1}^{*}\right)^{2}+q_{1}-\tau_{2} q_{0}\right) \sin \tau_{2} \omega_{1}^{*}\right] \sin \tau_{1}^{*} \omega_{1}^{*} \\
& +\left[n_{1}-3 n_{3}\left(\omega_{1}^{*}\right)^{2}+\left(2 q_{2}-\tau_{2} q_{1}\right) \omega_{1}^{*} \sin \tau_{2} \omega_{1}^{*}\right. \\
& \left.+\left(\tau_{2} q_{2}\left(\omega_{1}^{*}\right)^{2}+q_{1}-\tau_{2} q_{0}\right) \cos \tau_{2} \omega_{1}^{*}\right] \cos \tau_{1}^{*} \omega_{1}^{*} \\
& +\left[\left(2 p_{2}-\tau_{2} p_{1}\right) \omega_{1}^{*}+\tau_{2} p_{3}\left(\omega_{1}^{*}\right)^{3}\right] \sin \tau_{2} \omega_{1}^{*} \\
& +\left[p_{1}-\tau_{2} p_{0}-\left(3 p_{3}-\tau_{2} p_{2}\right)\left(\omega_{1}^{*}\right)^{2}\right] \cos \tau_{2} \omega_{1}^{*} \\
& +m_{1}-3 m_{3}\left(\omega_{1}^{*}\right)^{2}, \\
U_{42}= & {\left[2 n_{2} \omega_{1}^{*}+\left(2 q_{2}-\tau_{2} q_{1}\right) \omega_{1}^{*} \cos \tau_{2} \omega_{1}^{*}\right.} \\
& \left.-\left(\tau_{2} q_{2}\left(\omega_{1}^{*}\right)^{2}+q_{1}-\tau_{2} q_{0}\right) \sin \tau_{2} \omega_{1}^{*}\right] \cos \tau_{1}^{*} \omega_{1}^{*} \\
& -\left[n_{1}-3 n_{3}\left(\omega_{1}^{*}\right)^{2}+\left(2 q_{2}-\tau_{2} q_{1}\right) \omega_{1}^{*} \sin \tau_{2} \omega_{1}^{*}\right. \\
& \left.+\left(\tau_{2} q_{2}\left(\omega_{1}^{*}\right)^{2}+q_{1}-\tau_{2} q_{0}\right) \cos \tau_{2} \omega_{1}^{*}\right] \sin \tau_{1}^{*} \omega_{1}^{*} \\
& +\left[\left(2 p_{2}-\tau_{2} p_{1}\right) \omega_{1}^{*}+\tau_{2} p_{3}\left(\omega_{1}^{*}\right)^{3}\right] \cos \tau_{2} \omega_{1}^{*} \\
& -\left[p_{1}-\tau_{2} p_{0}-\left(3 p_{3}-\tau_{2} p_{2}\right)\left(\omega_{1}^{*}\right)^{2}\right] \sin \tau_{2} \omega_{1}^{*} \\
& +2 m_{2} \omega_{1}^{*}-4\left(\omega_{1}^{*}\right)^{3}, \\
V_{41}= & {\left[n_{0} \omega_{1}^{*}-n_{2}\left(\omega_{1}^{*}\right)^{3}+\left(q_{0} \omega_{1}^{*}-q_{2}\left(\omega_{1}^{*}\right)^{3}\right) \cos \tau_{2} \omega_{1}^{*}\right.} \\
& \left.+q_{1}\left(\omega_{1}^{*}\right)^{2} \sin \tau_{2} \omega_{1}^{*}\right] \sin \tau_{1}^{*} \omega_{1}^{*} \\
& +\left[n_{3}\left(\omega_{1}^{*}\right)^{4}-n_{1}\left(\omega_{1}^{*}\right)^{2}+\left(q_{0} \omega_{1}^{*}-q_{2}\left(\omega_{1}^{*}\right)^{3}\right) \sin \tau_{2} \omega_{1}^{*}\right. \\
& \left.-q_{1}\left(\omega_{1}^{*}\right)^{2} \cos \tau_{2} \omega_{1}^{*}\right] \cos \tau_{1}^{*} \omega_{1}^{*}, \\
V_{42}=[ & n_{0} \omega_{1}^{*}-n_{2}\left(\omega_{1}^{*}\right)^{3}+\left(q_{0} \omega_{1}^{*}-q_{2}\left(\omega_{1}^{*}\right)^{3}\right) \cos \tau_{2} \omega_{1}^{*} \\
& \left.+q_{1}\left(\omega_{1}^{*}\right)^{2} \sin \tau_{2} \omega_{1}^{*}\right] \cos \tau_{1}^{*} \omega_{1}^{*} \\
& -\left[n_{3}\left(\omega_{1}^{*}\right)^{4}-n_{1}\left(\omega_{1}^{*}\right)^{2}+\left(q_{0} \omega_{1}^{*}-q_{2}\left(\omega_{1}^{*}\right)^{3}\right) \sin \tau_{2} \omega_{1}^{*}\right. \\
& \left.-q_{1}\left(\omega_{1}^{*}\right)^{2} \cos \tau_{2} \omega_{1}^{*}\right] \sin \tau_{1}^{*} \omega_{1}^{*} \\
&
\end{aligned}
$$


Thus, if condition $\left(H_{42}\right): U_{41} V_{41}+U_{42} V_{42} \neq 0$ holds, then $\operatorname{Re}\left[\frac{d \lambda}{d \tau_{1}}\right]_{\tau_{1}=\tau_{1}^{*}}^{-1} \neq 0$. In conclusion, we have the following results.

Theorem 3 For system (3), suppose that conditions $\left(H_{1}\right),\left(H_{41}\right)$, and $\left(H_{42}\right)$ hold and $\tau_{2} \in$ $\left(0, \tau_{20}\right)$. Then system (3) is locally asymptotically stable when $\tau_{1} \in\left[0, \tau_{1}^{*}\right)$; system (3) undergoes a Hopf bifurcation at the worm-induced equilibrium $E_{*}$ when $\tau_{1}=\tau_{1}^{*}$, and a family of periodic solutions bifurcate from the worm-induced equilibrium $E_{*} ; \tau_{1}^{*}$ is defined as in Eq. (17).

Case $5 \tau_{2}>0, \tau_{1} \in\left(0, \tau_{10}\right)$. Let $\lambda=i \omega_{22}\left(\omega_{22}>0\right)$ be the root of Eq. (4). Then we can obtain

$$
\left\{\begin{array}{l}
M_{51}\left(\omega_{22}\right) \sin \tau_{2} \omega_{22}+M_{52}\left(\omega_{22}\right) \cos \tau_{2} \omega_{22}=M_{53}\left(\omega_{22}\right) \\
M_{51}\left(\omega_{22}\right) \cos \tau_{2} \omega_{22}-M_{52}\left(\omega_{22}\right) \sin \tau_{2} \omega_{22}=M_{54}\left(\omega_{22}\right)
\end{array}\right.
$$

where

$$
\begin{aligned}
M_{51}\left(\omega_{22}\right)= & p_{1} \omega_{22}-p_{3} \omega_{22}^{3}+q_{1} \omega_{22} \cos \tau_{1} \omega_{22}-\left(q_{0}-q_{2} \omega_{22}^{2}\right) \sin \tau_{1} \omega_{22} \\
M_{52}\left(\omega_{22}\right)= & p_{0}-p_{2} \omega_{22}^{2}+q_{1} \omega_{22} \sin \tau_{1} \omega_{22}+\left(q_{0}-q_{2} \omega_{22}^{2}\right) \cos \tau_{1} \omega_{22} \\
M_{53}\left(\omega_{22}\right)= & m_{2} \omega_{11}^{2}-\omega_{11}^{4}-m_{0}-\left(n_{1} \omega_{22}-n_{3} \omega_{22}^{3}\right) \sin \tau_{1} \omega_{22} \\
& -\left(p_{0}-p_{2} \omega_{11}^{2}\right) \cos \tau_{2} \omega_{11} \\
M_{54}\left(\omega_{11}\right)= & m_{3} \omega_{22}^{3}-m_{1} \omega_{22}-\left(n_{1} \omega_{22}-n_{3} \omega_{22}^{3}\right) \cos \tau_{1} \omega_{22} \\
& +\left(n_{0}-n_{2} \omega_{22}^{2}\right) \sin \tau_{1} \omega_{22}
\end{aligned}
$$

Thus we obtain the following equation with respect to $\omega_{22}$ :

$$
M_{53}^{2}\left(\omega_{22}\right)+M_{54}^{2}\left(\omega_{22}\right)-M_{51}^{2}\left(\omega_{22}\right)-M_{52}^{2}\left(\omega_{22}\right)=0 .
$$

Next, we assume that $\left(H_{51}\right)$ holds, that is, Eq. (18) has at least one positive root $\omega_{2}^{*}$. Thus Eq. (4) has a pair of purely imaginary roots $\pm i \omega_{2}^{*}$. For $\omega_{2}^{*}$, we have:

$$
\tau_{2}^{*}=\frac{1}{\omega_{2}^{*}} \times \arccos \left\{\frac{M_{51}\left(\omega_{1}^{*}\right) \times M_{54}\left(\omega_{1}^{*}\right)+M_{52}\left(\omega_{1}^{*}\right) \times M_{53}\left(\omega_{1}^{*}\right)}{M_{51}^{2}\left(\omega_{1}^{*}\right)+M_{52}^{2}\left(\omega_{1}^{*}\right)}\right\} .
$$

Similarly as in Case 4, we have

$$
\left[\frac{d \lambda}{d \tau_{2}}\right]^{-1}=\frac{P_{51}(\lambda)}{Q_{51}(\lambda)}-\frac{\tau_{2}}{\lambda}
$$

with

$$
\begin{aligned}
P_{51}(\lambda)= & 4 \lambda^{3}+3 m_{3} \lambda^{2}+2 m_{2} \lambda+m_{1}+\left(3 p_{3} \lambda^{2}+2 p_{2} \lambda+p_{1}\right) e^{-\lambda \tau_{2}} \\
& -\left[\tau_{1} n_{3} \lambda^{3}-\left(3 n_{3}-\tau_{1} n_{2}\right) \lambda^{2}-\left(2 n_{2}-\tau_{1} n_{1}\right) \lambda-n_{1}+\tau_{1} n_{0}\right] e^{-\lambda \tau_{1}} \\
& -\left[\tau_{1} q_{2} \lambda^{2}-\left(2 q_{2}-\tau_{1} q_{1}\right) \lambda-q_{1}+\tau_{1} q_{0}\right] e^{-\lambda\left(\tau_{1}+\tau_{2}\right)}, \\
Q_{51}(\lambda)= & \lambda\left(p_{3} \lambda^{3}+p_{2} \lambda^{2}+p_{1} \lambda+p_{0}\right) e^{-\lambda \tau_{2}}+\lambda\left(q_{2} \lambda^{2}+q_{1} \lambda+q_{0}\right) e^{-\lambda\left(\tau_{1}+\tau_{2}\right)} .
\end{aligned}
$$


Similarly as in Case 4, we can obtain

$$
\operatorname{Re}\left[\frac{d \lambda}{d \tau_{2}}\right]_{\tau_{2}=\tau_{2}^{*}}^{-1}=\frac{U_{51} V_{51}+U_{52} V_{52}}{V_{51}^{2}+V_{52}^{2}},
$$

where

$$
\begin{aligned}
U_{51}= & {\left[2 p_{2} \omega_{2}^{*}+\left(2 q_{2}-\tau_{1} q_{1}\right) \omega_{2}^{*} \cos \tau_{1} \omega_{2}^{*}-\left(\tau_{1} q_{2}\left(\omega_{2}^{*}\right)^{2}+q_{1}-\tau_{1} q_{0}\right) \sin \tau_{1} \omega_{2}^{*}\right] \sin \tau_{2}^{*} \omega_{2}^{*} } \\
& +\left[p_{1}-3 p_{3}\left(\omega_{2}^{*}\right)^{2}+\left(2 q_{2}-\tau_{1} q_{1}\right) \omega_{2}^{*} \sin \tau_{1} \omega_{2}^{*}\right. \\
& \left.+\left(\tau_{1} q_{2}\left(\omega_{2}^{*}\right)^{2}+q_{1}-\tau_{2} q_{0}\right) \cos \tau_{1} \omega_{2}^{*}\right] \cos \tau_{2}^{*} \omega_{2}^{*} \\
& +\left[\left(2 n_{2}-\tau_{1} n_{1}\right) \omega_{2}^{*}+\tau_{1} n_{3}\left(\omega_{2}^{*}\right)^{3}\right] \sin \tau_{1} \omega_{2}^{*} \\
& +\left[n_{1}-\tau_{1} n_{0}-\left(3 n_{3}-\tau_{1} n_{2}\right)\left(\omega_{2}^{*}\right)^{2}\right] \cos \tau_{2} \omega_{2}^{*}, \\
U_{52}= & {\left[2 p_{2} \omega_{2}^{*}+\left(2 q_{2}-\tau_{1} q_{1}\right) \omega_{2}^{*} \cos \tau_{1} \omega_{2}^{*}-\left(\tau_{1} q_{2}\left(\omega_{2}^{*}\right)^{2}+q_{1}-\tau_{1} q_{0}\right) \sin \tau_{1} \omega_{2}^{*}\right] \cos \tau_{2}^{*} \omega_{2}^{*} } \\
& -\left[p_{1}-3 p_{3}\left(\omega_{2}^{*}\right)^{2}+\left(2 q_{2}-\tau_{1} q_{1}\right) \omega_{2}^{*} \sin \tau_{1} \omega_{2}^{*}\right. \\
& \left.+\left(\tau_{1} q_{2}\left(\omega_{2}^{*}\right)^{2}+q_{1}-\tau_{1} q_{0}\right) \cos \tau_{1} \omega_{2}^{*}\right] \sin \tau_{2}^{*} \omega_{2}^{*} \\
& +\left[\left(2 n_{2}-\tau_{1} n_{1}\right) \omega_{2}^{*}+\tau_{1} n_{3}\left(\omega_{2}^{*}\right)^{3}\right] \cos \tau_{1} \omega_{2}^{*} \\
& -\left[n_{1}-\tau_{1} n_{0}-\left(3 n_{3}-\tau_{1} n_{2}\right)\left(\omega_{2}^{*}\right)^{2}\right] \sin \tau_{1} \omega_{2}^{*}, \\
V_{51}= & {\left[p_{0} \omega_{2}^{*}-p_{2}\left(\omega_{2}^{*}\right)^{3}+\left(q_{0} \omega_{2}^{*}-q_{2}\left(\omega_{2}^{*}\right)^{3}\right) \cos \tau_{1} \omega_{2}^{*}+q_{1}\left(\omega_{2}^{*}\right)^{2} \sin \tau_{1} \omega_{2}^{*}\right] \sin \tau_{2}^{*} \omega_{2}^{*} } \\
& +\left[p_{3}\left(\omega_{2}^{*}\right)^{4}-p_{1}\left(\omega_{2}^{*}\right)^{2}+\left(q_{0} \omega_{2}^{*}-q_{2}\left(\omega_{2}^{*}\right)^{3}\right) \sin \tau_{1} \omega_{2}^{*}-q_{1}\left(\omega_{2}^{*}\right)^{2} \cos \tau_{1} \omega_{2}^{*}\right] \cos \tau_{2}^{*} \omega_{2}^{*}, \\
V_{52}= & {\left[p_{0} \omega_{2}^{*}-p_{2}\left(\omega_{2}^{*}\right)^{3}+\left(q_{0} \omega_{2}^{*}-q_{2}\left(\omega_{2}^{*}\right)^{3}\right) \cos \tau_{1} \omega_{2}^{*}+q_{1}\left(\omega_{2}^{*}\right)^{2} \sin \tau_{1} \omega_{2}^{*}\right] \cos \tau_{2}^{*} \omega_{2}^{*} } \\
& -\left[p_{3}\left(\omega_{2}^{*}\right)^{4}-p_{1}\left(\omega_{2}^{*}\right)^{2}+\left(q_{0} \omega_{2}^{*}-q_{2}\left(\omega_{2}^{*}\right)^{3}\right) \sin \tau_{1} \omega_{2}^{*}-q_{1}\left(\omega_{2}^{*}\right)^{2} \cos \tau_{1} \omega_{2}^{*}\right] \sin \tau_{2}^{*} \omega_{2}^{*} .
\end{aligned}
$$

Thus, if condition

$\left(H_{52}\right): U_{51} V_{51}+U_{52} V_{52} \neq 0$

holds, then $\operatorname{Re}\left[\frac{d \lambda}{d \tau_{2}}\right]_{\tau_{2}=\tau_{2}^{*}}^{-1} \neq 0$. In conclusion, we have the following results.

Theorem 4 For system (3), suppose that conditions $\left(H_{1}\right),\left(H_{51}\right)$, and $\left(H_{52}\right)$ hold and $\tau_{1} \in$ $\left(0, \tau_{10}\right)$. Then system (3) is locally asymptotically stable when $\tau_{2} \in\left[0, \tau_{2}^{*}\right)$; system (3) undergoes a Hopf bifurcation at the worm-induced equilibrium $E_{*}$ when $\tau_{2}=\tau_{2}^{*}$, and a family of periodic solutions bifurcate from the worm-induced equilibrium $E_{*} ; \tau_{2}^{*}$ is defined as in Eq. (19).

\section{Direction and stability of Hopf bifurcation}

Following the idea of Hassard [34], in this section, we investigate the direction and stability of the Hopf bifurcation at the critical value $\tau_{2}^{*}$ by using the normal form theory and the center manifold theorem. Throughout this section, we assume that $\tau_{1 *}<\tau_{1}^{*}$, where $\tau_{1 *} \in\left(0, \tau_{10}\right)$. Let $\tau_{2}=\tau_{2}^{*}+\mu(\mu \in \mathrm{R}), u_{1}=S\left(\tau_{2} t\right), u_{2}=E_{1}\left(\tau_{2} t\right), u_{3}=E_{2}\left(\tau_{2} t\right)$, and $u_{4}=I\left(\tau_{2} t\right)$. System (3) becomes

$$
\dot{u}(t)=L_{\mu}\left(u_{t}\right)+F\left(\mu, u_{t}\right)
$$


where $u(t)=\left(u_{1}, u_{2}, u_{3}, u_{4}\right)^{T} \in C=C\left([-1,0], \mathrm{R}^{4}\right)$, and $L_{\mu}: C \rightarrow \mathrm{R}^{4}$ and $F: \mathrm{R} \times C \rightarrow \mathrm{R}^{4}$ are defined as

$$
L_{\mu} \phi=\left(\tau_{2}^{*}+\mu\right)\left(A_{\max } \phi(0)+B_{\max } \phi\left(-\frac{\tau_{1 *}}{\tau_{2}^{*}}\right)+C_{\max } \phi(-1)\right)
$$

and

$$
F(\mu, \phi)=\left(\tau_{2}^{*}+\mu\right)\left[\begin{array}{c}
-\beta \phi_{1}(0) \phi_{4}(0) \\
p \beta \phi_{1}(0) \phi_{4}(0) \\
q \beta \phi_{1}(0) \phi_{4}(0) \\
0
\end{array}\right]
$$

with

$$
\begin{aligned}
A_{\max } & =\left(\begin{array}{cccc}
\alpha_{11} & 0 & 0 & \alpha_{14} \\
\alpha_{21} & \alpha_{22} & 0 & \alpha_{24} \\
\alpha_{31} & 0 & \alpha_{33} & \alpha_{34} \\
0 & 0 & 0 & \alpha_{44}
\end{array}\right), \quad B_{\max }=\left(\begin{array}{cccc}
0 & 0 & 0 & 0 \\
0 & \beta_{22} & 0 & 0 \\
0 & 0 & 0 & 0 \\
0 & \beta_{42} & 0 & 0
\end{array}\right), \\
C_{\max } & =\left(\begin{array}{cccc}
0 & 0 & 0 & 0 \\
0 & 0 & 0 & 0 \\
0 & 0 & \gamma_{33} & 0 \\
0 & 0 & \gamma_{43} & 0
\end{array}\right) .
\end{aligned}
$$

Thus by the Reisz representation theorem there exists $\eta(\theta, \mu)$ such that

$$
L_{\mu} \phi=\int_{-1}^{0} d \eta(\theta, \mu) \phi(\theta), \quad \text { for } \phi \in C .
$$

In fact, we can choose

$$
\eta(\theta, \mu)= \begin{cases}\left(\tau_{2}^{*}+\mu\right)\left(A_{\max }+B_{\max }+C_{\max }\right), & \theta=0, \\ \left(\tau_{2}^{*}+\mu\right)\left(B_{\max }+C_{\max }\right), & \theta \in\left[-\frac{\tau_{1 *}}{\tau_{2}^{*}}, 0\right), \\ \left(\tau_{2}^{*}+\mu\right) C_{\max }, & \theta \in\left(-1,-\frac{\tau_{1 *}}{\tau_{2}^{*}}\right), \\ 0, & \theta=-1,\end{cases}
$$

where $\delta(\theta)$ is the Dirac delta function.

For $\phi \in C\left([-1,0], \mathrm{R}^{4}\right)$, define

$$
A(\mu) \phi= \begin{cases}\frac{d \phi(\theta)}{d \theta}, & -1 \leq \theta<0, \\ \int_{-1}^{0} d \eta(\theta, \mu) \phi(\theta), & \theta=0,\end{cases}
$$

and

$$
R(\mu) \phi= \begin{cases}0, & -1 \leq \theta<0, \\ F(\mu, \phi), & \theta=0 .\end{cases}
$$




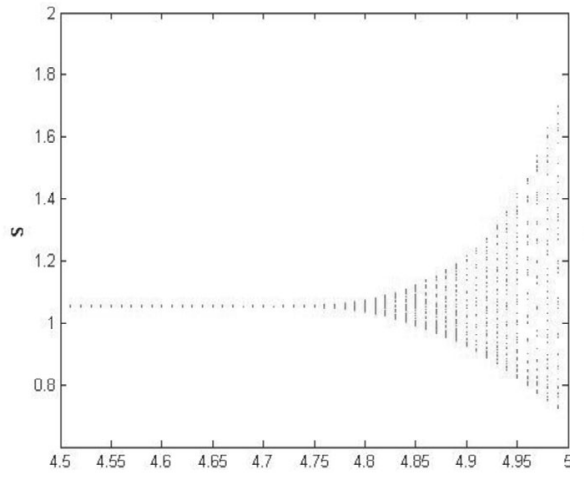

(a)

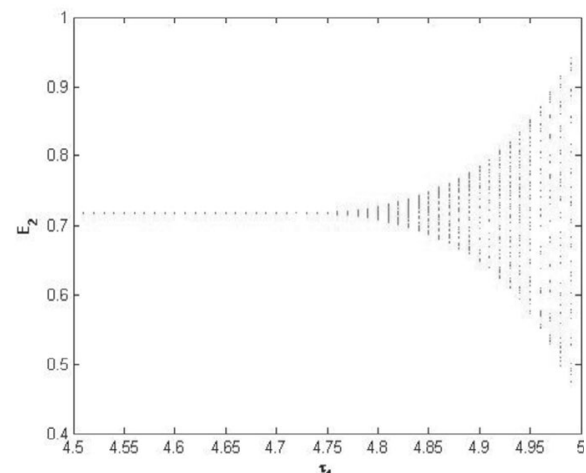

(c)

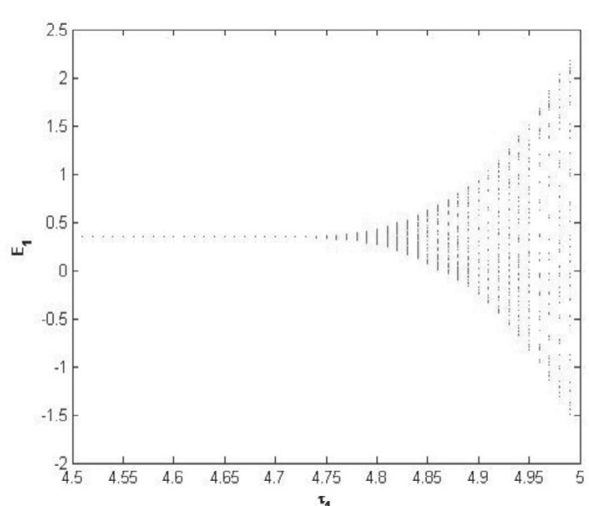

(b)

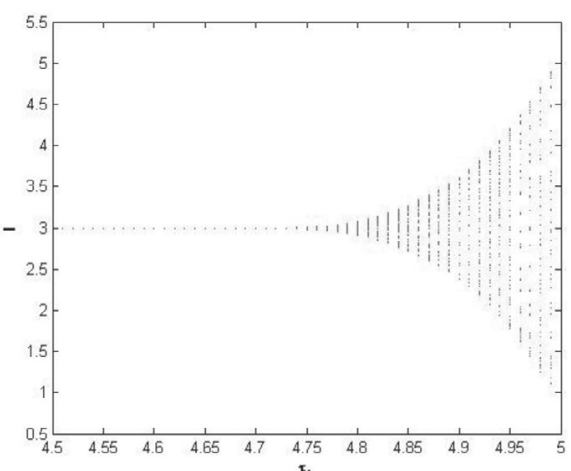

(d)

Figure 1 The bifurcation diagram with respect to $\tau_{1}$ when $\tau_{2}=0$

Then system (20) is equivalent to

$$
\dot{u}(t)=A(\mu) u_{t}+R(\mu) u_{t} .
$$

For $\varphi \in C^{1}\left([0,1],\left(\mathrm{R}^{4}\right)^{*}\right)$, define

$$
A^{*}(\varphi)= \begin{cases}-\frac{d \varphi(s)}{d s}, & 0<s \leq 1, \\ \int_{-1}^{0} d \eta^{T}(s, 0) \varphi(-s), & s=0,\end{cases}
$$

and the bilinear inner form for $A$ and $A^{*}$

$$
\langle\varphi(s), \phi(\theta)\rangle=\bar{\varphi}(0) \phi(0)-\int_{\theta=-1}^{0} \int_{\xi=0}^{\theta} \bar{\varphi}(\xi-\theta) d \eta(\theta) \phi(\xi) d \xi
$$

where $\eta(\theta)=\eta(\theta, 0)$.

Let $\rho(\theta)=\left(1, \rho_{2}, \rho_{3}, \rho_{4}\right)^{T} e^{i \tau_{2}^{*} \omega_{2}^{*} \theta}$ be the eigenvector of $A(0)$ corresponding to $+i \tau_{2}^{*} \omega_{2}^{*}$, and let $\rho^{*}(s)=D\left(1, \rho_{2}^{*}, \rho_{3}^{*}, \rho_{4}^{*}\right)^{T} e^{i \tau_{2}^{*} \omega_{2}^{*} s}$ be the eigenvector of $A^{*}(0)$ corresponding to $-i \tau_{2}^{*} \omega_{2}^{*}$. By the definition of $A(0)$ and $A^{*}$ we get

$$
\rho_{2}=\frac{\alpha_{21}+\alpha_{24} \rho_{4}}{i \omega_{2}^{*}-\alpha_{22}-\beta_{22} e^{-i \tau_{1 *} \omega_{2}^{*}}},
$$




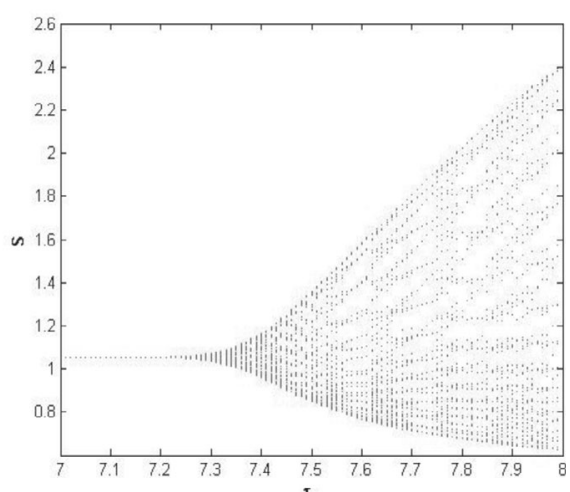

(a)

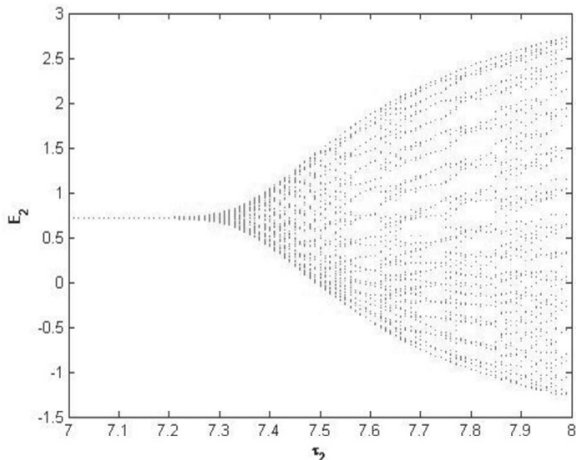

(c)

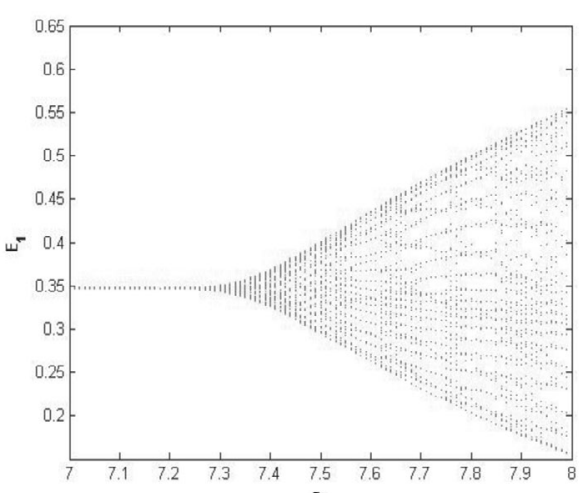

(b)

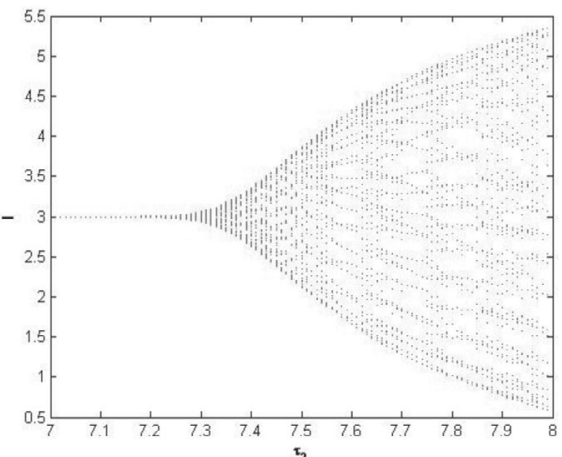

(d)

Figure 2 The bifurcation diagram with respect to $\tau_{2}$ when $\tau_{1}=0$

$$
\begin{aligned}
& \rho_{3}=\frac{\left(i \omega_{2}^{*}-\alpha_{44}\right) \rho_{4}-\beta_{42} e^{-i \tau_{1 *} \omega_{2}^{*}} \rho_{2}}{\gamma_{43} e^{-i \tau_{2}^{*} \omega_{2}^{*}}}, \\
& \rho_{4}=\frac{i \omega_{2}^{*}-\alpha_{11}}{\alpha_{14}}, \\
& \rho_{2}^{*}=\rho_{41}^{*} \rho_{4}^{*}, \quad \rho_{3}^{*}=\rho_{42}^{*} \rho_{4}^{*}, \\
& \rho_{4}^{*}=\frac{\alpha_{14}}{\rho_{41}^{*}+\rho_{42}^{*}-i \omega_{2}^{*}}, \\
& \rho_{41}^{*}=-\frac{\beta_{42} e^{i \tau_{1 *} \omega_{2}^{*}}}{i \omega_{2}^{*}+\alpha_{22}+\beta_{22} e^{i \tau_{1 *} \omega_{2}^{*}}}, \\
& \rho_{42}^{*}=-\frac{\gamma_{43} e^{i \tau_{2}^{*} \omega_{2}^{*}}}{i \omega_{2}^{*}+\alpha_{33}+\gamma_{33} e^{i \tau_{2}^{*} \omega_{2}^{*}} .}
\end{aligned}
$$

From Eq. (24) the expression of $Q$ can be obtained as follows:

$$
\begin{aligned}
\bar{D}= & {\left[1+\rho_{2} \bar{\rho}_{2}^{*}+\rho_{3} \bar{\rho}_{3}^{*}+\rho_{4} \bar{\rho}_{4}^{*}+\tau_{1 *} e^{-i \tau_{1 *} \omega_{2}^{*}} \rho_{2}\left(\beta_{22} \bar{\rho}_{2}^{*}+\beta_{42} \bar{\rho}_{4}^{*}\right)\right.} \\
& \left.+\tau_{2}^{*} e^{-i \tau_{2}^{*} \omega_{2}^{*}} \rho_{3}\left(\gamma_{33} \bar{\rho}_{3}^{*}+\gamma_{43} \bar{\rho}_{4}^{*}\right)\right]^{-1},
\end{aligned}
$$

where $\left\langle\rho^{*}, \rho\right\rangle=1$ and $\left\langle\rho^{*}, \bar{\rho}\right\rangle=0$. 


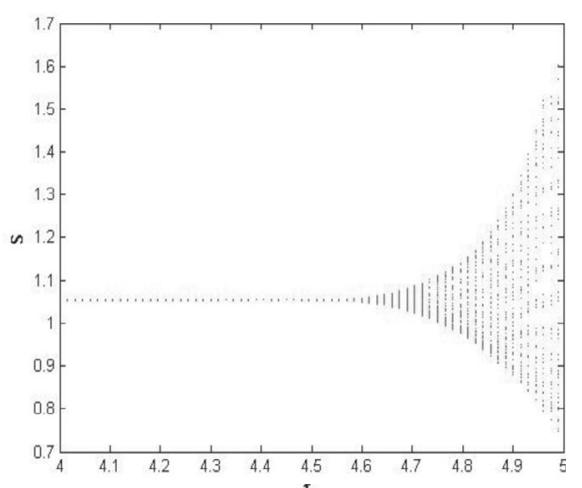

(a)

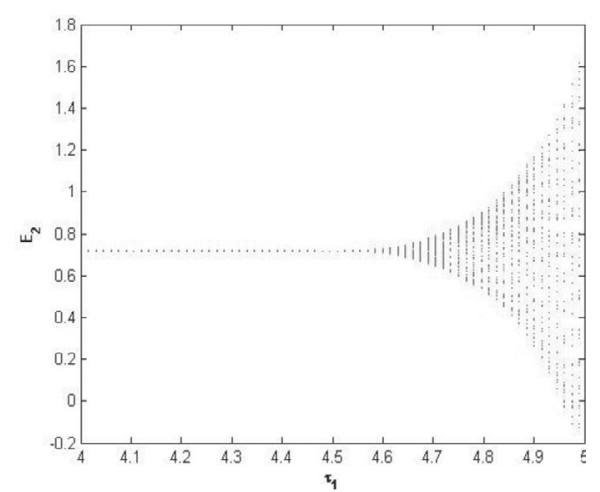

(c)

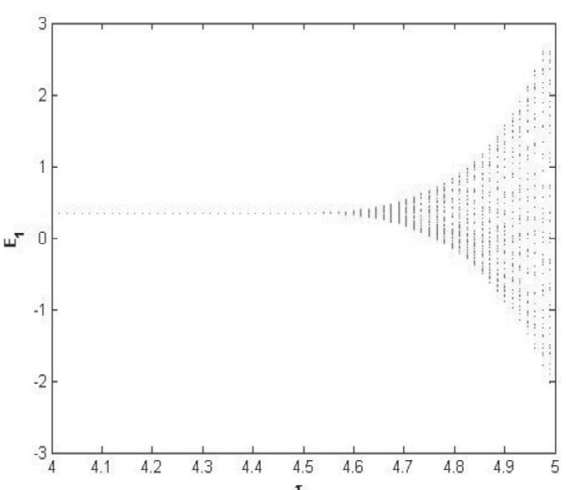

(b)

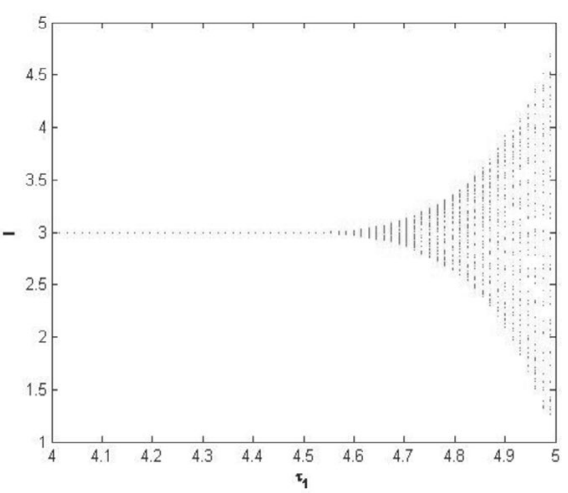

(d)

Figure 3 The bifurcation diagram with respect to $\tau_{1}$ when $\tau_{2}=4.25 \in\left(0, \tau_{20}\right)$

In what follows, we can obtain $g_{20}, g_{11}, g_{02}$, and $g_{21}$ by using the algorithms in [34] and a similar computation process as that in $[29,35,36]$ :

$$
\begin{aligned}
g_{20}= & 2 \tau_{2}^{*} \bar{D} \beta \rho_{4}\left(p \bar{\rho}_{2}^{*}+q \bar{\rho}_{3}^{*}-1\right), \\
g_{11}= & \tau_{2}^{*} \bar{D} \beta \operatorname{Re}\left\{\rho_{4}\right\}\left(p \bar{\rho}_{2}^{*}+q \bar{\rho}_{3}^{*}-1\right), \\
g_{02}= & 2 \tau_{2}^{*} \bar{D} \beta \bar{\rho}_{4}\left(p \bar{\rho}_{2}^{*}+q \bar{\rho}_{3}^{*}-1\right), \\
g_{21}= & 2 \tau_{2}^{*} \bar{D} \beta\left(p \bar{\rho}_{2}^{*}+q \bar{\rho}_{3}^{*}-1\right)\left(W_{11}^{(1)}(0) \rho_{4}\right. \\
& \left.+\frac{1}{2} W_{20}^{(1)}(0) \bar{\rho}_{4}+W_{11}^{(4)}(0)+\frac{1}{2} W_{20}^{(4)}(0)\right),
\end{aligned}
$$

with

$$
\begin{aligned}
& W_{20}(\theta)=\frac{i g_{20} \rho(0)}{\tau_{2}^{*} \omega_{2}^{*}} e^{i \tau_{2}^{*} \omega_{2}^{*} \theta}+\frac{i \bar{g}_{02} \bar{\rho}(0)}{3 \tau_{2}^{*} \omega_{2}^{*}} e^{-i \tau_{2}^{*} \omega_{2}^{*} \theta}+E_{1} e^{2 i \tau_{2}^{*} \omega_{2}^{*} \theta}, \\
& W_{11}(\theta)=-\frac{i g_{11} \rho(0)}{\tau_{2}^{*} \omega_{2}^{*}} e^{i \tau_{2}^{*} \omega_{2}^{*} \theta}+\frac{i \bar{g}_{11} \bar{\rho}(0)}{\tau_{2}^{*} \omega_{2}^{*}} e^{-i \tau_{2}^{*} \omega_{2}^{*} \theta}+E_{2} .
\end{aligned}
$$




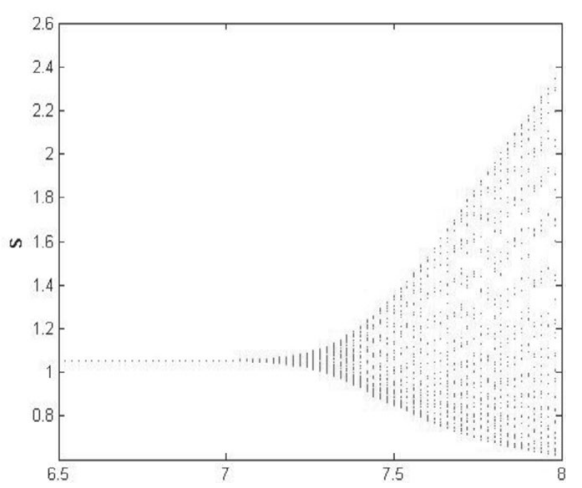

(a)

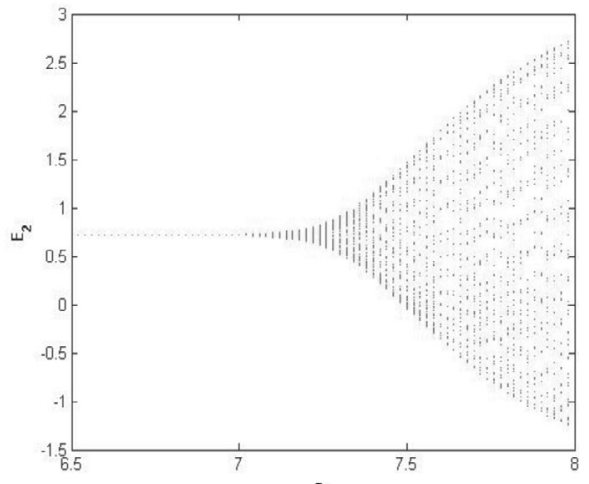

(c)

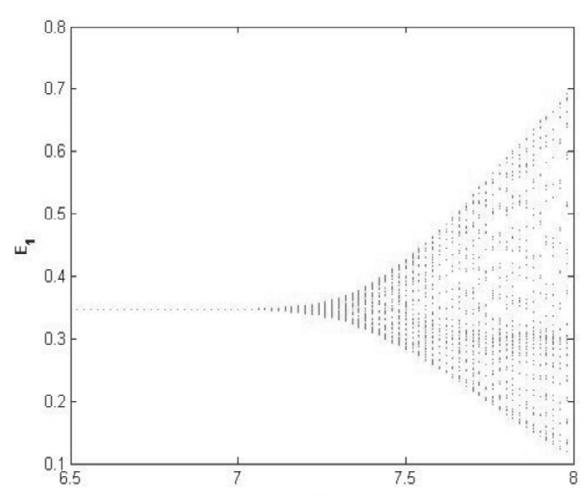

(b)

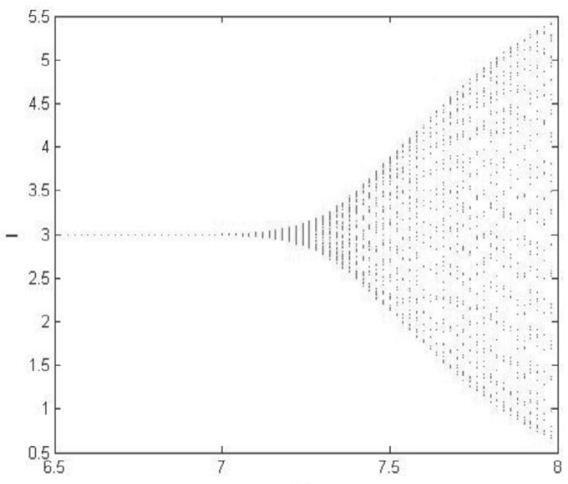

(d)

Figure 4 The bifurcation diagram with respect to $\tau_{2}$ when $\tau_{1}=2.25 \in\left(0, \tau_{10}\right)$

$E_{1}$ and $E_{2}$ can be obtained by the following two equations:

$$
\begin{aligned}
& E_{1}=2\left(\begin{array}{cccc}
2 i \omega_{2}^{*}-\alpha_{11} & 0 & 0 & -\alpha_{14} \\
-\alpha_{21} & 2 i \omega_{0}-\alpha_{22}-\beta_{22} e^{-2 i \tau_{1 *} \omega_{2}^{*}} & 0 & -\alpha_{24} \\
-\alpha_{31} & 0 & 2 i \omega_{2}^{*}-\alpha_{33}-\gamma_{33} e^{-2 i \tau_{2}^{*} \omega_{2}^{*}} & -\alpha_{34} \\
0 & -\beta_{42} e^{-2 i \tau_{1 *} \omega_{2}^{*}} & -\gamma_{43} e^{-2 i \tau_{2}^{*} \omega_{2}^{*}} & 2 i \omega_{2}^{*}-\alpha_{44}
\end{array}\right)^{-1} \\
& \times\left(\begin{array}{c}
-\beta \rho_{4} \\
p \beta \rho_{4} \\
q \beta \rho_{4} \\
0
\end{array}\right) \\
& E_{2}=-\left(\begin{array}{cccc}
\alpha_{11} & 0 & 0 & -\alpha_{14} \\
\alpha_{21} & \alpha_{22}+\beta_{22} & 0 & \alpha_{24} \\
\alpha_{31} & 0 & \alpha_{33}+\gamma_{33} & \alpha_{34} \\
0 & \beta_{42} & \gamma_{43} & \alpha_{44}
\end{array}\right)^{-1} \times\left(\begin{array}{c}
-\beta \operatorname{Re}\left\{\rho_{4}\right\} \\
p \beta \operatorname{Re}\left\{\rho_{4}\right\} \\
q \beta \operatorname{Re}\left\{\rho_{4}\right\} \\
0
\end{array}\right) .
\end{aligned}
$$




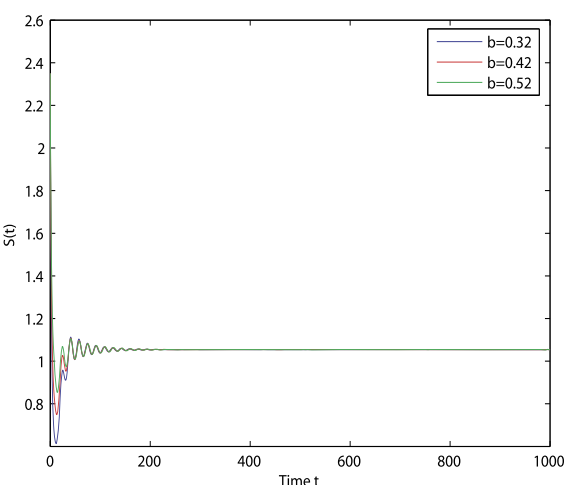

(a)

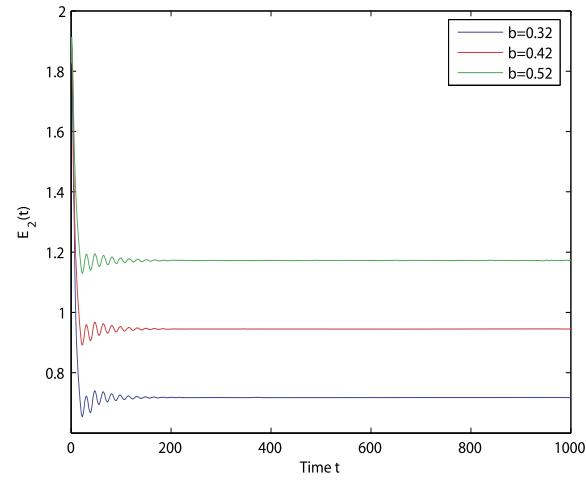

(c)

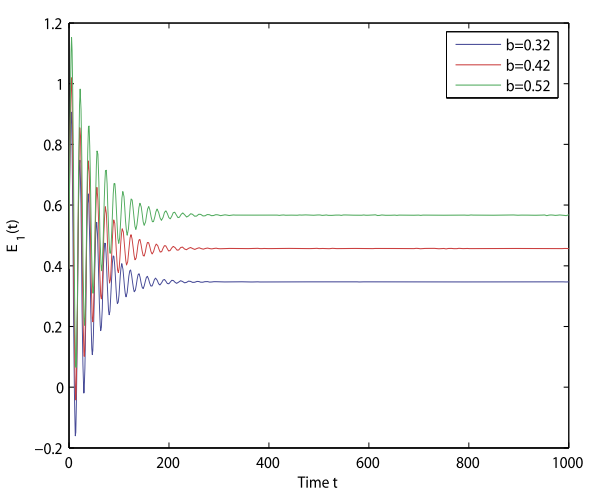

(b)

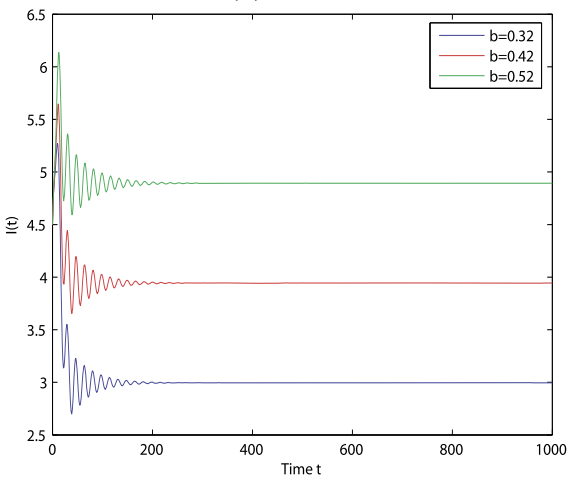

(d)

Figure 5 Time plots of $S, E_{1} E_{2}$ and $/$ for different $b$ at $\tau_{1}=4.25<\tau_{10}$ when $\tau_{2}=0$. Rest of the parameters are taken as given in the text

Figure 6 Dynamic behavior of system (26): projection on $S-E_{1}-/$ with $\tau_{1}=4.75<\tau_{10}$ when $\tau_{2}=0$ for different $b$. Rest of the parameters are taken as given in the text

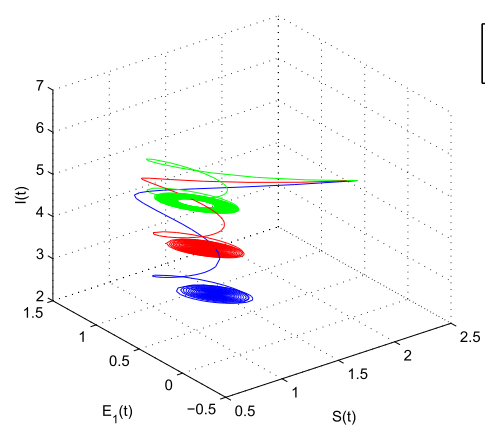

Then we can obtain

$$
\begin{aligned}
& C_{1}(0)=\frac{i}{2 \tau_{2}^{*} \omega_{2}^{*}}\left(g_{11} g_{20}-2\left|g_{11}\right|^{2}-\frac{\left|g_{02}\right|^{2}}{3}\right)+\frac{g_{21}}{2} \\
& \mu_{2}=-\frac{\operatorname{Re}\left\{C_{1}(0)\right\}}{\operatorname{Re}\left\{\lambda^{\prime}\left(\tau_{2}^{*}\right)\right\}} \\
& \beta_{2}=2 \operatorname{Re}\left\{C_{1}(0)\right\} \\
& T_{2}=-\frac{\operatorname{Im}\left\{C_{1}(0)\right\}+\mu_{2} \operatorname{Im}\left\{\lambda^{\prime}\left(\tau_{2}^{*}\right)\right\}}{\tau_{2}^{*} \omega_{2}^{*}} .
\end{aligned}
$$




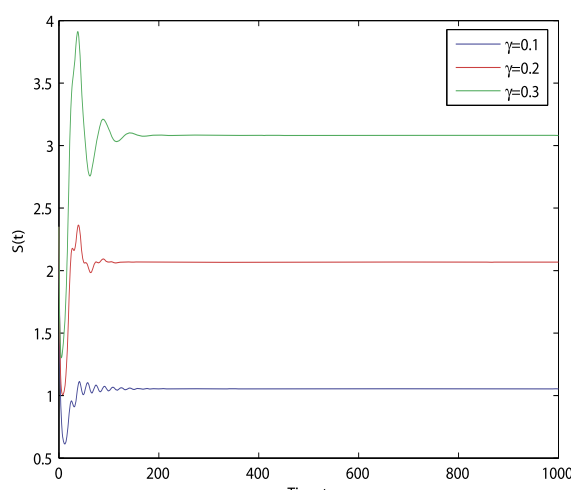

(a)

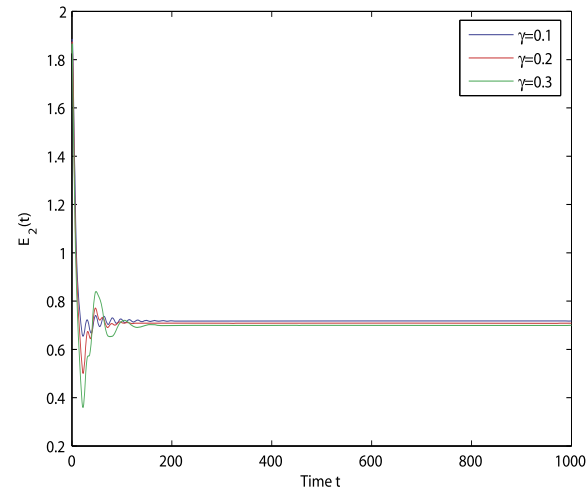

(c)

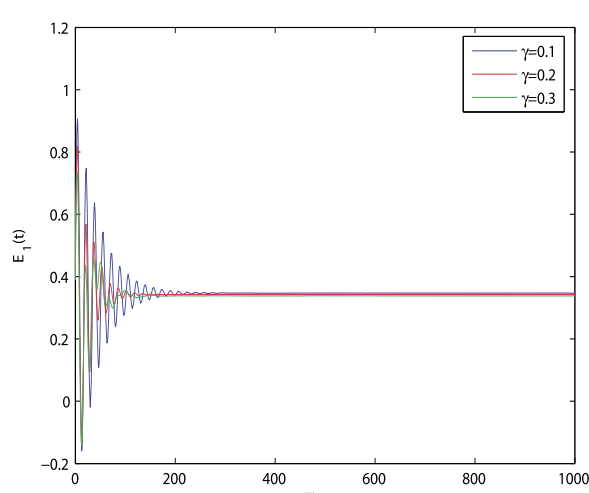

(b)

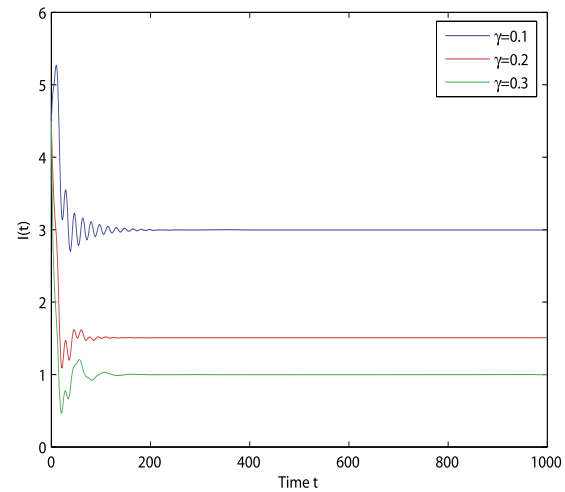

(d)

Figure 7 Time plots of $S, E_{1} E_{2}$ and / for different $\gamma$ at $\tau_{1}=4.25$ when $\tau_{2}=0$. Rest of the parameters are taken as given in the text

Figure 8 Dynamic behavior of system (26): projection on $S-E_{1}-/$ with $\tau_{1}=4.95>\tau_{10}$ when $\tau_{2}=0$ for different $\gamma$. Rest of the parameters are taken as given in the text

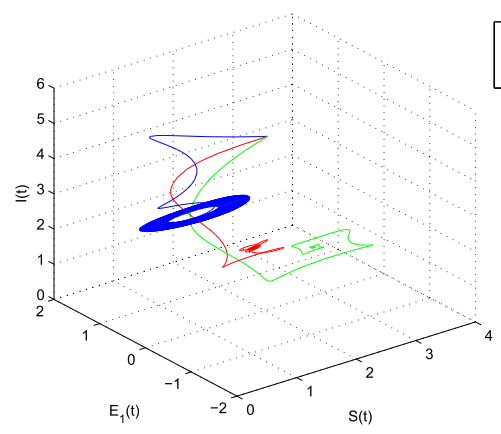

In conclusion, we have the following results.

Theorem 5 For system (2), if $\mu_{2}>0\left(\mu_{2}<0\right)$, then the Hopf bifurcation is supercritical (subcritical); if $\beta_{2}<0\left(\beta_{2}>0\right)$, then the bifurcating periodic solutions are stable (unstable); if $T_{2}>0\left(T_{2}<0\right)$, then the period of the bifurcating periodic solutions increase (decrease).

\section{Numerical simulations}

For verifying accuracy and correctness of the obtained theoretical results, in this section, we execute some numerical simulations. For simulation, we choose the following set of 


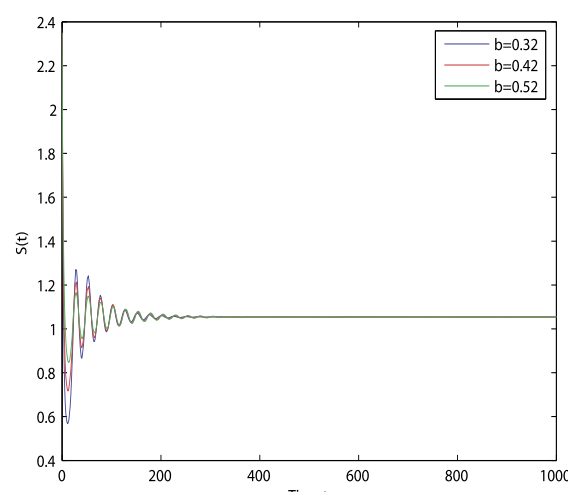

(a)

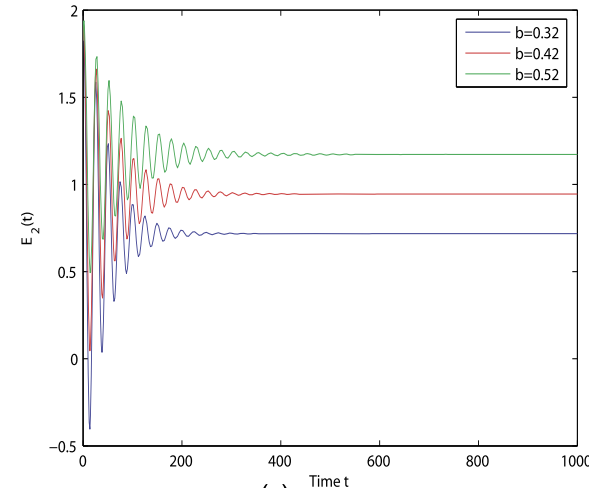

(c)

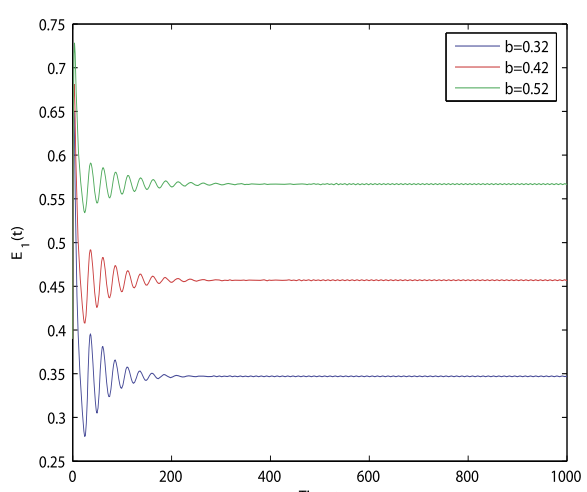

(b)

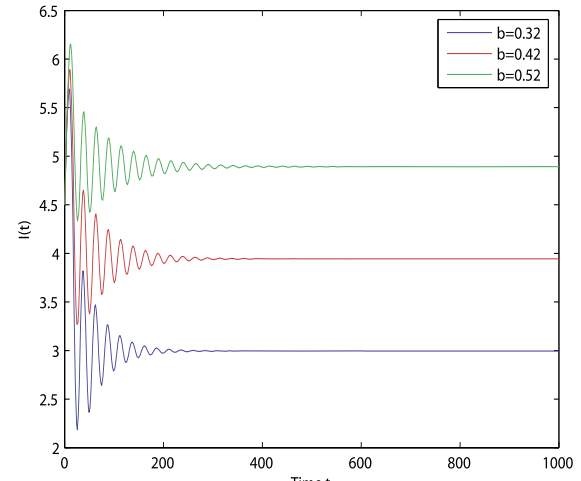

(d)

Figure 9 Time plots of $S_{1} E_{1}, E_{2}$ and $/$ for different $b$ at $\tau_{2}=6.25<\tau_{20}$ when $\tau_{1}=0$. Rest of the parameters are taken as given in the text

Figure 10 Dynamic behavior of system (26): projection on $S-E_{1}-/$ with $\tau_{2}=6.25<\tau_{20}$ when $\tau_{1}=0$ for different $b$. Rest of the parameters are taken as given in the text

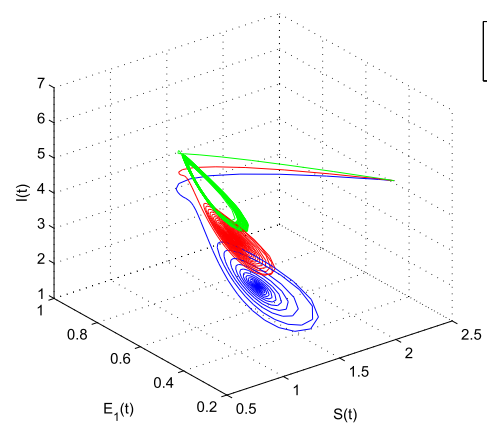

parameters: $b=0.32, \beta=0.1, \sigma=0.004, p=0.4, q=0.6, \alpha_{1}=0.36, \alpha_{2}=0.26, \gamma=0.1$. Then we obtain the following specific case of system (3):

$$
\left\{\begin{array}{l}
\frac{d S(t)}{d t}=0.32-0.1 S(t) I(t)-0.004 S(t), \\
\frac{d E_{1}(t)}{d t}=0.04 S(t) I(t)-0.004 E_{1}(t)-0.36 E_{1}\left(t-\tau_{1}\right), \\
\frac{d E_{2}(t)}{d t}=0.06 S(t) I(t)-0.004 E_{2}(t)-0.26 E_{2}\left(t-\tau_{2}\right), \\
\frac{d I(t)}{d t}=0.36 E_{1}\left(t-\tau_{1}\right)+0.26 E_{2}\left(t-\tau_{2}\right)-0.104 I(t) .
\end{array}\right.
$$




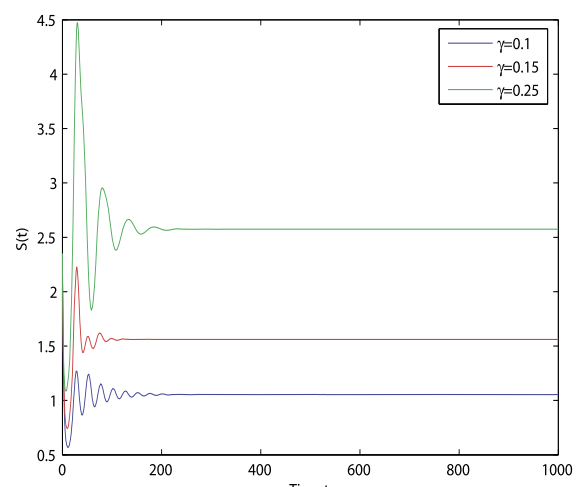

(a)

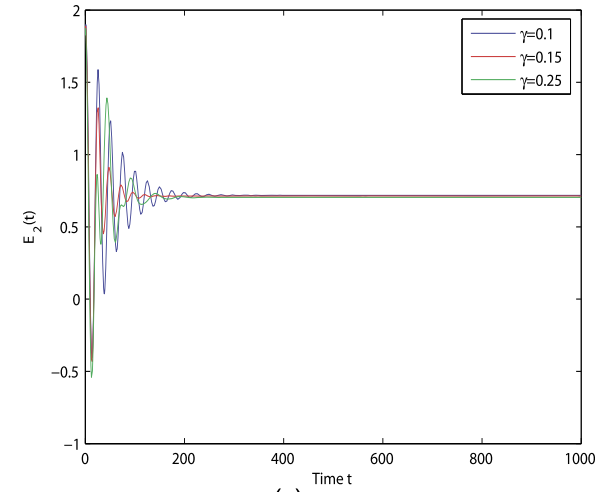

(c)

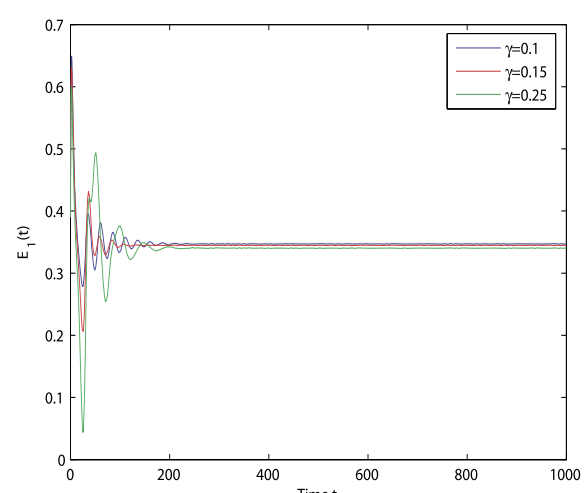

(b)

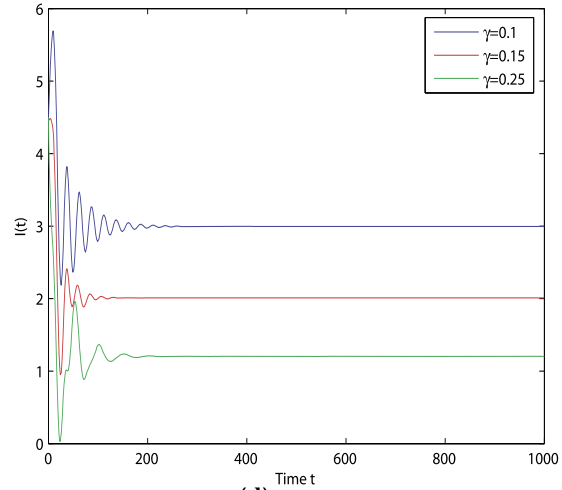

(d)

Figure 11 Time plots of $S, E_{1}, E_{2}$ and / for different $\gamma$ at $\tau_{2}=6.25$ when $\tau_{1}=0$. Rest of the parameters are taken as given in the text

Figure 12 Dynamic behavior of system (26): projection on $S-E_{1}-/$ with $\tau_{2}=7.55>\tau_{20}$ when $\tau_{1}=0$ for different $\gamma$. Rest of the parameters are taken as given in the text

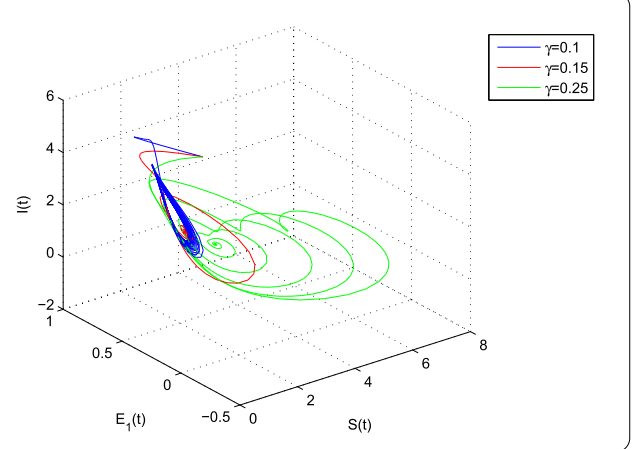

With the help of Matlab package software we obtain $R_{0}=76.7543$. Further, we obtain the unique worm-induced equilibrium $E_{*}(1.0542,0.3470,0.7177,2.9954)$. Now we can validate for the worm-induced equilibrium that $m_{10}=0.003>0, m_{12} m_{13}=0.3785>m_{11}=$ 0.0377 , and $m_{11} m_{12} m_{13}=0.1368>m_{12}^{2}+m_{10} m_{13}^{2}=0.0143$ by means of Matlab software package. So system (26) is locally asymptotically stable in absence of delay.

Further, by some complex computations we can obtain $\omega_{10}=0.2907$ and $\tau_{10}=4.7657$ when $\tau_{2}=0 ; \omega_{20}=0.7062$ and $\tau_{20}=7.2185$ when $\tau_{1}=0 ; \omega_{1}^{*}=1.4981$ and $\tau_{1}^{*}=4.5750$ when $\tau_{2}=4.25 \in\left(0, \tau_{20}\right)$; and $\omega_{2}^{*}=0.0067$ and $\tau_{2}^{*}=7.0505$ when $\tau_{1}=2.25 \in\left(0, \tau_{10}\right)$. It follows from Theorems $1-4$ that the worm-induced equilibrium $E_{*}(1.0542,0.3470,0.7177,2.9954)$ 


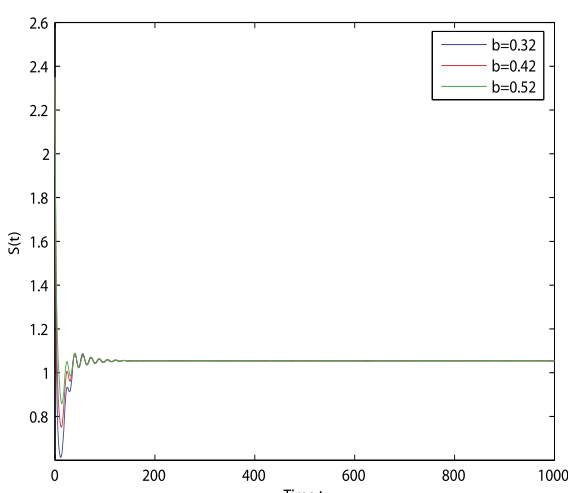

(a)

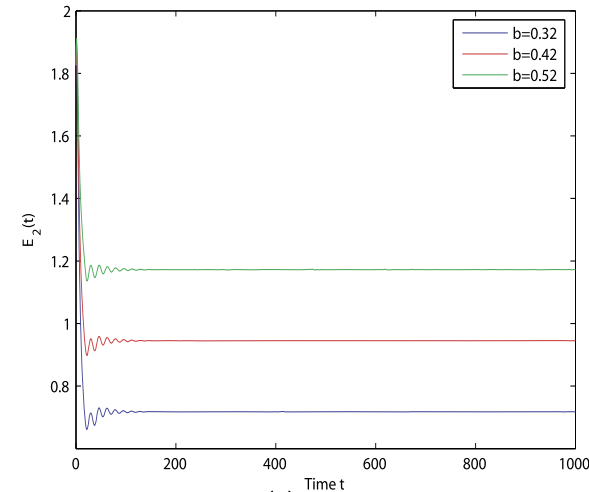

(c)

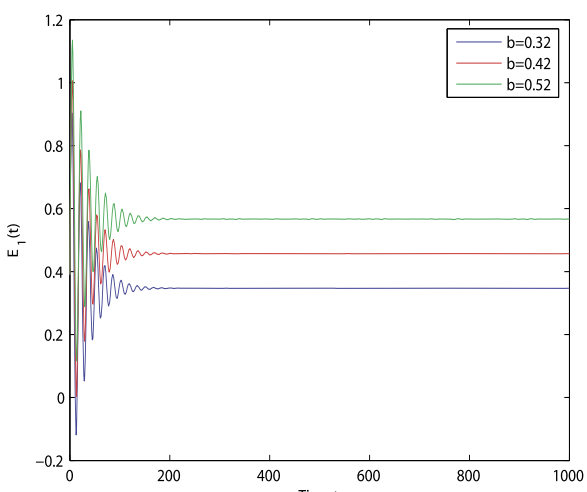

(b) Time

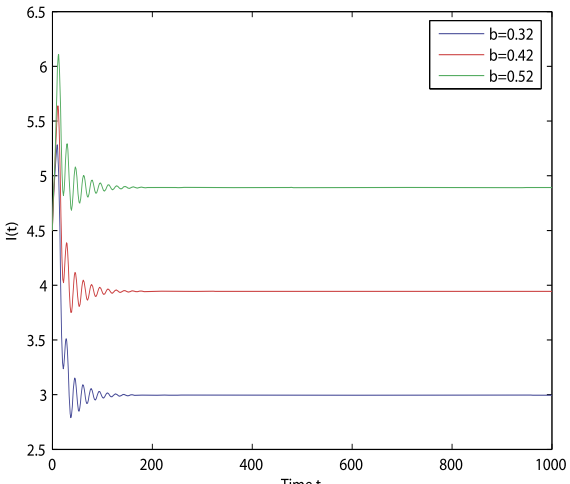

(d)

Figure 13 Time plots of $S, E_{1}, E_{2}$ and $/$ for different $b$ at $\tau_{1}=4.05$ when $\tau_{2}=4.25 \in\left(0, \tau_{20}\right)$. Rest of the parameters are taken as given in the text

Figure 14 Dynamic behavior of system (26): projection on $S-E_{1}-/$ with $\tau_{1}=4.75>\tau_{1}^{*}$ when $\tau_{2}=4.25 \in\left(0, \tau_{20}\right)$ for different $b$. Rest of the parameters are taken as given in the text

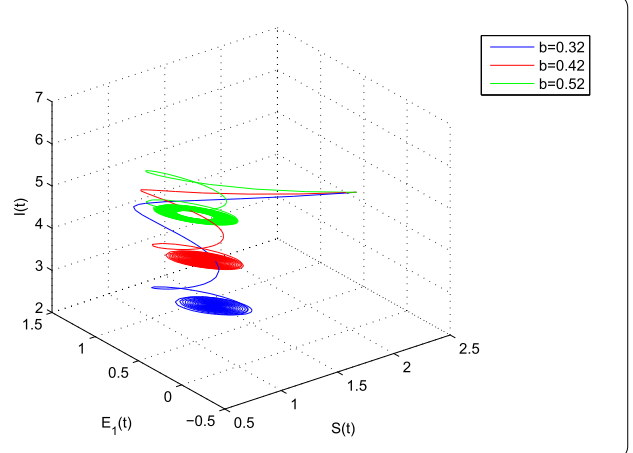

is locally asymptotically stable when the value of the time delay is under the critical value, and in this case the propagation of the worms can be controlled. However, the worminduced equilibrium $E_{*}(1.0542,0.3470,0.7177,2.9954)$ will lose its stability, and a Hopf bifurcation will occur once the delay passes through the critical value, and in this case the propagation of the worms will be out of control. This property can be illustrated by the bifurcation diagrams shown in Figs. 1-4.

Figure $5(\mathrm{a}-\mathrm{d})$ demonstrate the effect of the constant recruitment to susceptible nodes $b$ on system dynamics and show that the infected class of short latent period, the infected class of long latent period, and the infectious class increases, whereas the susceptible class 


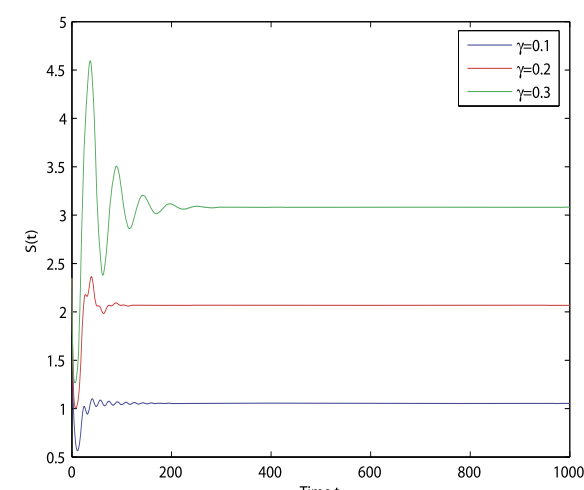

(a)

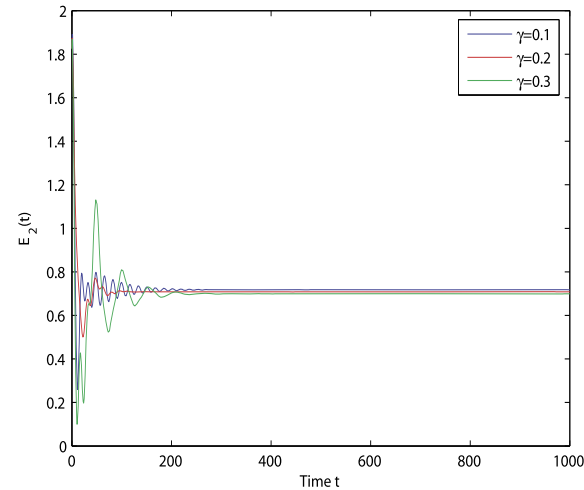

(c)

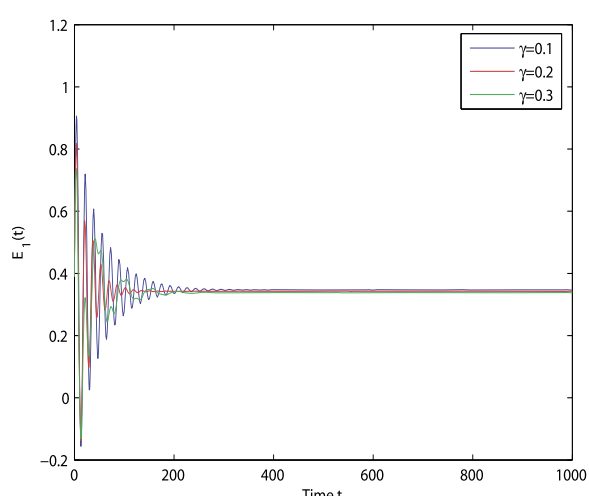

(b)

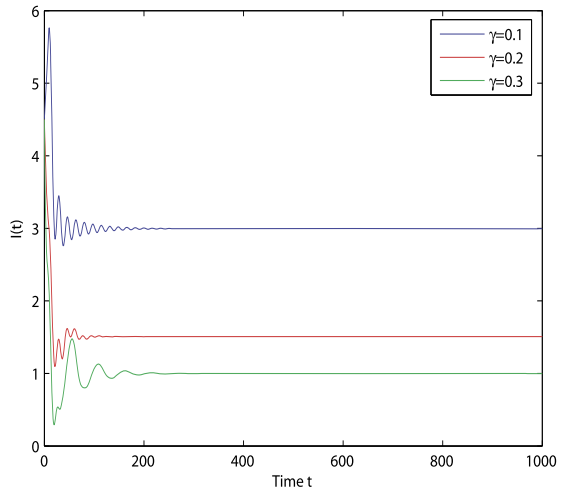

(d)

Figure 15 Time plots of $S, E_{1}, E_{2}$ and / for different $\gamma$ at $\tau_{1}=4.05$ when $\tau_{2}=4.25 \in\left(0, \tau_{20}\right)$. Rest of the parameters are taken as given in the text

Figure 16 Dynamic behavior of system (26): projection on $S-E_{1}-/$ with $\tau_{1}=4.75>\tau_{1}^{*}$ when $\tau_{2}=4.25 \in\left(0, \tau_{20}\right)$ for different $\gamma$. Rest of the

parameters are taken as given in the text

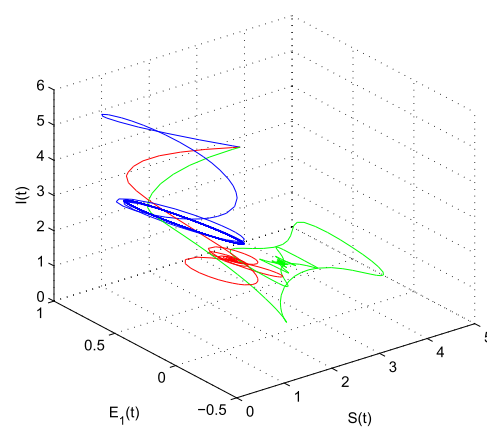

keeps static along with the increment of $b$ when $\tau_{1}>0$ and $\tau_{2}=0$. We can also observe that oscillations and delay can be reduced and removed by decreasing the value of $b$, which is illustrated by Fig. 6. Figure $7(\mathrm{a}-\mathrm{d})$ describe the effect of the recovery rate of the infectious nodes $\gamma$ on system dynamics and show that the susceptible class increases, whereas the infected class of short latent period, the infected class of long latent period, and the infectious class decreases when $\tau_{1}>0$ and $\tau_{2}=0$. Also, oscillations and delay can be reduced and removed by increasing the value of $\gamma$, which is illustrated by Fig. 8. The effects of $b$ and $\gamma$ on system dynamics when $\tau_{1}=0$ and $\tau_{2}>0, \tau_{1}>0$ and $\tau_{2} \in\left(0, \tau_{20}\right)$, and $\tau_{2}>0$ and $\tau_{1} \in\left(0, \tau_{10}\right)$ are the same as those in the case where $\tau_{1}>0$ and $\tau_{2}=0$. The simulations are 


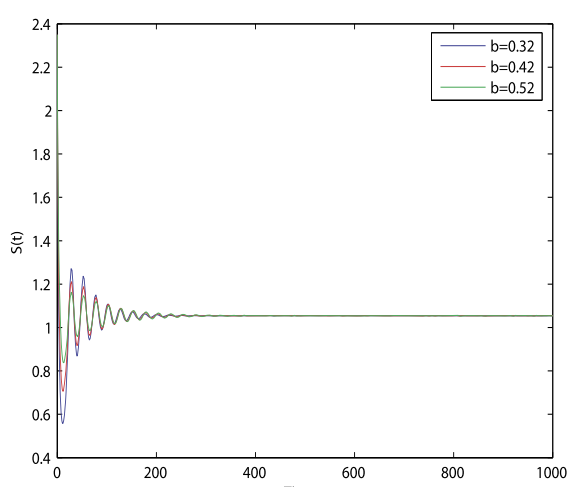

(a)

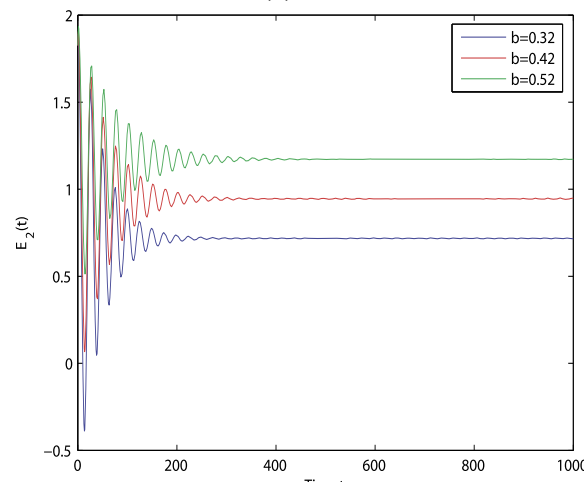

(c)

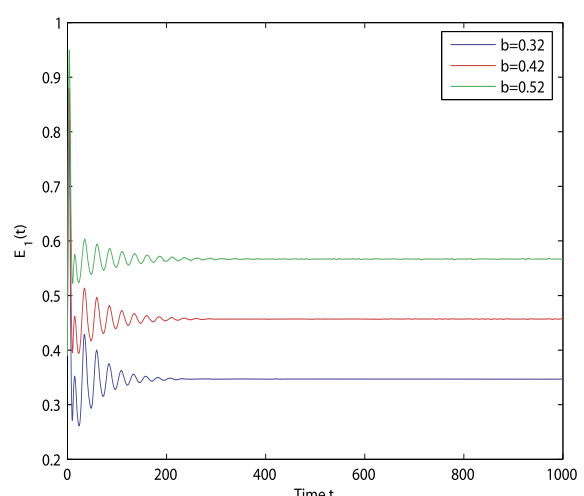

(b)

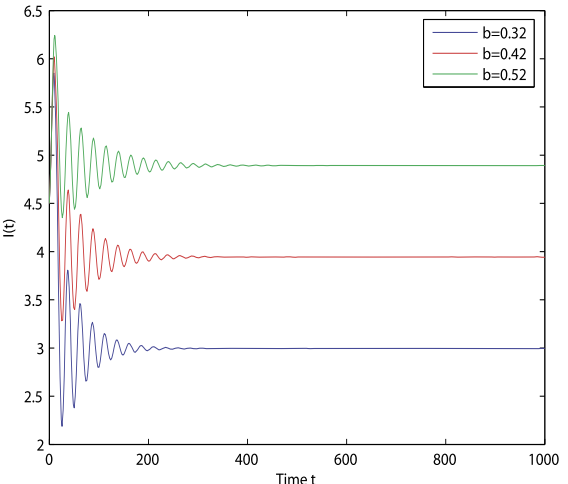

(d)

Figure 17 Time plots of $S, E_{1}, E_{2}$ and $/$ for different $b$ at $\tau_{2}=6.25$ when $\tau_{1}=2.25 \in\left(0, \tau_{10}\right)$. Rest of the parameters are taken as given in the text

Figure 18 Dynamic behavior of system (26): projection on $S-E_{1}-/$ with $\tau_{2}=6.95>\tau_{2}^{*}$ when $\tau_{1}=2.25 \in\left(0, \tau_{10}\right)$ for different $b$. Rest of the parameters are taken as given in the text

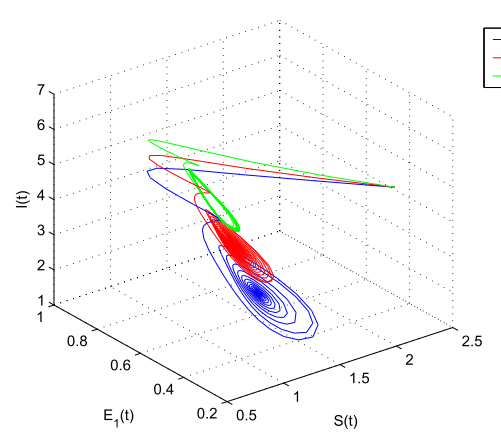

shown in Figs. 9-20. Thus we can conclude that the constant recruitment to susceptible nodes $b$ and the recovery rate of the infectious nodes $\gamma$ have a tremendous effect on the system dynamics.

\section{Conclusions}

In the present paper, we investigated a delayed epidemic model for the propagation of worm in wireless sensor network with two latent periods by incorporating the latent delays into the formulated model in the literature [25] considering the typical latent feature of the malicious codes in networks. We mainly consider the effect of the latent delay on the proposed model. In comparison with the other worm propagation models with time delay, 


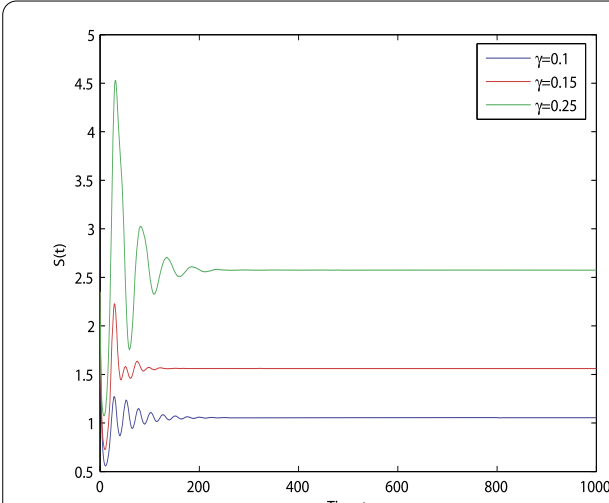

(a)

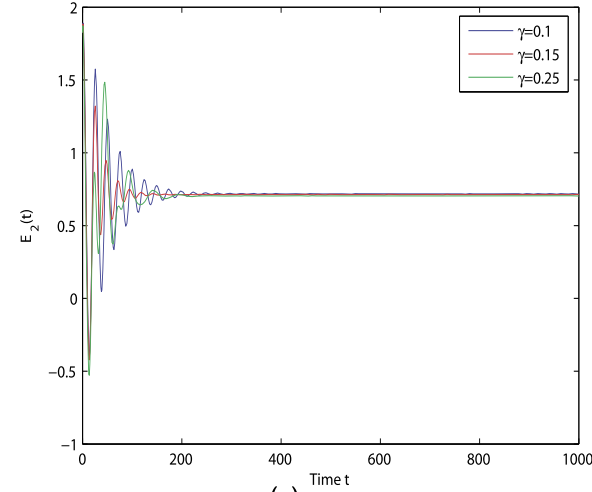

(c)

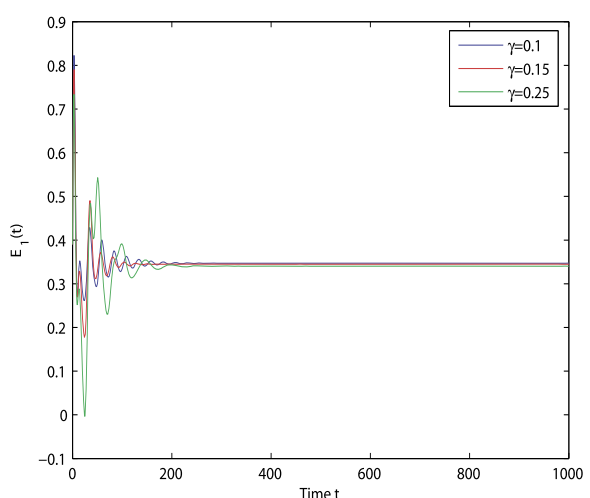

(b)

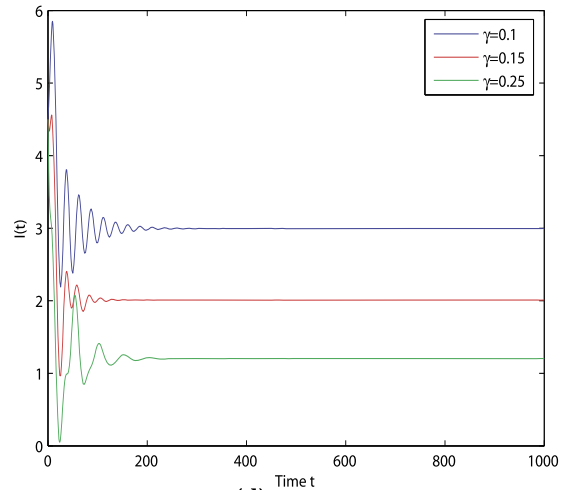

(d)

Figure 19 Time plots of $S, E_{1}, E_{2}$ and $/$ for different $\gamma$ at $\tau_{2}=6.25$ when $\tau_{1}=2.25 \in\left(0, \tau_{10}\right)$. Rest of the parameters are taken as given in the text

Figure 20 Dynamic behavior of system (26): projection on $S-E_{1}-/$ with $\tau_{2}=7.55>\tau_{2}^{*}$ when $\tau_{1}=2.25 \in\left(0, \tau_{10}\right)$ for different $\gamma$. Rest of the parameters are taken as given in the text

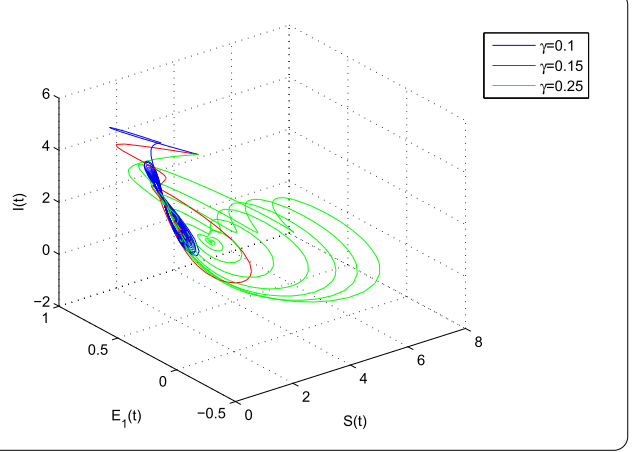

in the proposed model, we considered different types of worms in the wireless sensor network. Thus we can conclude that the model investigated is more general and overcomes the insufficiency of the existing worm models to a certain extent.

The local stability and existence of Hopf bifurcation at the worm-induced equilibrium are investigated, and the threshold values of Hopf bifurcation are obtained by satisfying transversality conditions for showing the delay dynamics of the work in [25]. We numerically demonstrated that the propagation of the worms in the wireless sensor network can be controlled when the values of the latent delays are below the threshold value. However, the propagation of the worms is out of control when the values of the latent delays pass through the threshold value. We can conclude that the time delay should 
be controlled below the threshold value, particularly, the direction and stability of the Hopf bifurcation when $\tau_{2}>0$ and $\tau_{1} \in\left(0, \tau_{10}\right)$. By numerical simulations we have $C_{1}(0)=$ $-0.003182-i 0.000519, \mu_{2}=43.589041>0, \beta_{2}=-0.006364<0$, and $T_{2}=-0.034226<0$ when $\tau_{2}>0$ and $\tau_{1 *}=2.25 \in\left(0, \tau_{10}\right)$. Therefore by Theorem 5 we can deduce that the Hopf bifurcation is supercritical and the bifurcating periodic solutions are stable with decreasing period. Since the bifurcating periodic solutions are stable, the numbers of every class of sensor nodes in system (26) may coexist in an oscillatory mode. This phenomenon is not welcome in the wireless sensor networks. According to the numerical simulations, we can see that the onset of the Hopf bifurcation and the oscillation can be delayed if the values of the constant recruitment to susceptible nodes $b$ and the recovery rate of the infectious nodes $\gamma$ change properly. Thus we strongly recommend that the managers of the wireless sensor network should properly control the constant recruitment to susceptible nodes and update the antivirus software timely to control the propagation of worms in the wireless sensor network easily.

\section{Acknowledgements}

The authors are grateful to the editor and the anonymous referees for their valuable comments and suggestions on the paper.

\section{Funding}

This work was supported by University Top-notch Scholarship Program of Anhui Province (gxbjZD49), Bengbu University National Research Fund Cultivation Project (2017GJPY03), Natural Science Foundation of Inner Mongolia Autonomous Region (No. 2018MS01023), and Project of Support Program for Excellent Youth Talent in Colleges and Universities of Anhui Province (No. gxyqZD2018044).

Availability of data and materials

The authors declare that all the data can be accessed in the numerical simulation section of our manuscript.

Competing interests

The authors declare that there is no conflict of interests.

Authors' contributions

Both authors read and approved the final manuscript.

\section{Author details}

${ }^{1}$ School of Science, Bengbu University, Bengbu, China. ${ }^{2}$ School of Management Science and Engineering, Anhui University of Finance and Economics, Bengbu, China.

\section{Publisher's Note}

Springer Nature remains neutral with regard to jurisdictional claims in published maps and institutional affiliations.

Received: 23 June 2019 Accepted: 2 October 2019 Published online: 17 October 2019

\section{References}

1. Singh, J., Kumar, D., Hammouch, Z., Atangana, A.: A fractional epidemiological model for computer viruses pertaining to a new fractional derivative. Appl. Math. Comput. 316, 504-515 (2018)

2. Zhao, T., Bi, D.J.: Hopf bifurcation of a computer virus spreading model in the network with limited anti-virus ability. Adv. Differ. Equ. 2018, Article ID 183 (2018)

3. Ren, J.G., Yang, X.F., Zhu, Q.Y., Yang, L.X., Zhang, C.M.: A novel computer virus model and its dynamics. Nonlinear Anal., Real World Appl. 13, 376-384 (2012)

4. Keshri, N., Mishra, B.K.: Two time-delay dynamic model on the transmission of malicious signals in wireless sensor network. Chaos Solitons Fractals 68, 151-158 (2014)

5. Fu, X.W., Yang, Y.S., Yao, H.Q.: Analysis on invulnerability of wireless sensor network towards cascading failures based on coupled map lattice. Complexity 2018, Article ID 6386324 (2018)

6. Ren, J.G., Xu, Y.H.: A compartmental model for computer virus propagation with kill signals. Physica A 486, 446-454 (2017)

7. Yang, L.X., Yang, X.F., Tang, Y.Y.: A bi-virus competing spreading model with generic infection rates. IEEE Trans. Netw. Sci. Eng. 5, 2-12 (2018)

8. Tang, S., Mark, B.L.: Analysis of virus spread in wireless sensor networks: an epidemic model. In: 7th International Workshop on the Design of Reliable Communication Networks (2009)

9. Feng, L.P., Song, L.P., Zhao, Q.S., Wang, H.B.: Modeling and stability analysis of worm propagation in wireless sensor network. Math. Probl. Eng. 2015, Article ID 129598 (2015) 
10. Zhu, L.H., Zhao, H.Y., Wang, X.M.: Bifurcation analysis of a delay reaction-diffusion malware propagation model with feedback control. Commun. Nonlinear Sci. Numer. Simul. 22, 747-768 (2015)

11. Mishra, B.K., Keshri, N.: Mathematical model on the transmission of worms in wireless sensor network. Appl. Math. Model. 37, 4103-4111 (2013)

12. Upadhyay, R.K., Kumari, S.: Detecting malicious chaotic signals in wireless sensor network. Physica A 492, 1129-1152 (2018)

13. Singh, A., Awasthi, A.K., Singh, K., Srivastava, P.K.: Modeling and analysis of worm propagation in wireless sensor networks. Wirel. Pers. Commun. 98, 2535-2551 (2018)

14. Nwokoye, C.H., Ejiofor, V.E., Orji, R.: Investigating the effect of uniform random distribution of nodes in wireless sensor networks using an epidemic worm model. In: The International Conference on Computing Research and Innovations, Ibadan, Nigeria (2016)

15. Zhang, Z.Z., Si, F.S.: Dynamics of a delayed SEIRS-V model on the transmission of worms in a wireless sensor network. Adv. Differ. Equ. 2014, Article ID 295 (2014)

16. Ojha, R.P., Sanyal, G., Srivastava, P.K., Sharma, K.: Design and analysis of modified SIQRS model for performance study of wireless sensor network. Scalable Comp. Pract. Exp. 18, 229-241 (2017)

17. Khanh, N.H.: Dynamics of a worm propagation model with quarantine in wireless sensor networks. Appl. Math. Inf. Sci. 10, 1739-1746 (2016)

18. Xiao, X., Fu, P., Dou, C.S., Li, Q., Hu, G.W., Xia, S.T.: Design and analysis of SElQR worm propagation model in mobile Internet. Commun. Nonlinear Sci. Numer. Simul. 43, 341-350 (2017)

19. Nwokoye, C.H., Ozoegwu, G.C., Ejiofor, V.E.: Pre-quarantine approach for defense against propagation of malicious objects in networks. Int. J. Comput. Netw. Inf. Secur. 2, 43-52 (2017)

20. Nwokoye, C.H., Umeh, I.I. The SEIQR-V model: on a more accurate analytical characterization of malicious threat defense. Int. J. Inf. Technol. Comput. Sci. 12, 28-37 (2017)

21. Ojha, R.P., Srivastava, P.K., Awasthi, S., Sanyal, G.: Global stability of dynamic model for worm propagation in wireless sensor network. In: Proceeding of International Conference on Intelligent Communication, Control and Devices. Adv. Intel. Sys. Comput., vol. 479, pp. 695-703 (2017)

22. Keshri, A.K., Mishra, B.K., Mallick, D.K.: A predator-prey model on the attacking behavior of malicious objects in wireless nanosensor networks. Nano Commun. Netw. 15, 1-16 (2018)

23. Srivastava, A.P., Awasthi, S., Ojha, R.P., Srivastava, P.K., Katiyar, S.: Stability analysis of SIDR model for worm propagation in wireless sensor network. Indian J. Sci. Technol. 9, 1-5 (2016)

24. Upadhyay, R.K., Kumari, S.: Bifurcation analysis of an e-epidemic model in wireless sensor network. Int. J. Comput. Math. 95, 1775-1805 (2018)

25. Ojha, R.P., Srivastava, P.K., Sanyal, G.: Mathematical model for wireless sensor network with two latent periods. In: Next-Generation Networks. Adv. Intel. Sys. Comput., vol. 638, pp. 497-504 (2018)

26. Ren, J.G., Yang, X.F., Yang, X.L., Xu, Y.H., Yang, F.Z.: A delayed computer virus propagation model and its dynamics. Chaos Solitons Fractals 45, 74-79 (2012)

27. Soumenn, K., Sarit, M.: Dynamics of a delayed predator-prey system with stage structure and cooperation for preys. Chaos Solitons Fractals 114, 453-460 (2018)

28. Zhang, X.B., Zhu, H.L.: Hopf bifurcation and chaos of a delayed finance system. Complexity 2019, Article ID 6715036 (2019)

29. Jana, D., Agrawal, R., Upadhyay, R.K.: Top-predator interference and gestation delay as determinants of the dynamics of a realistic model food chain. Chaos Solitons Fractals 69, 50-63 (2014)

30. Sun, X.G., Wei, J.J.: Stability and bifurcation analysis in a viral infection model with delays. Adv. Differ. Equ. 2015, Article ID 332 (2015)

31. Kundu, S., Maitra, S.: Dynamical behaviour of a delayed three species predator-prey model with cooperation among the prey species. Nonlinear Dyn. 92, 627-643 (2018)

32. Liu, J., Wang, K.: Hopf bifurcation of a delayed SIQR epidemic model with constant input and nonlinear incidence rate. Adv. Differ. Equ. 2016, Article ID 168 (2016)

33. Li, X.L., Wei, J.J.: On the zeros of a fourth degree exponential polynomial with applications to a neural network model with delays. Chaos Solitons Fractals 26, 519-526 (2005)

34. Hassard, B.D., Kazarinoff, N.D., Wan, Y.H.: Theory and Applications of Hopf Bifurcation. Cambridge University Press, Cambridge (1981)

35. Gori, L., Guerrini, L., Sodini, M.: Hopf bifurcation in a cobweb model with discrete time delays. Discrete Dyn. Nat. Soc. 2014, Article ID 137090 (2014)

36. Upadhyay, R.K., Agrawal, R.: Dynamics and responses of a predator-prey system with competitive interference and time delay. Nonlinear Dyn. 83, 821-837 (2016) 